\title{
Length Scales in Brownian yet Non-Gaussian Dynamics
}

\author{
José M. Miotto®, ${ }^{1}$ Simone Pigolotti $\odot,{ }^{2}$ Aleksei V. Chechkin, ${ }^{3,4}$ and Sándalo Roldán-Vargas $\odot^{5,6, *}$ \\ ${ }^{1}$ Leiden Institute of Advanced Computer Science, 2333 CA Leiden, Netherlands \\ ${ }^{2}$ Biological Complexity Unit, Okinawa Institute for Science and Technology and Graduate University, \\ Onna, Okinawa 904-0495, Japan \\ ${ }^{3}$ Institute for Physics and Astronomy, University of Potsdam, 14476 Potsdam-Golm, Germany \\ ${ }^{4}$ Akhiezer Institute for Theoretical Physics, Kharkov 61108, Ukraine \\ ${ }^{5}$ Max Planck Institute for the Physics of Complex Systems, 01187 Dresden, Germany \\ ${ }^{6}$ Department of Applied Physics, Faculty of Sciences, University of Granada, 18071 Granada, Spain
}

(Received 25 September 2020; revised 12 March 2021; accepted 12 May 2021; published 2 July 2021; corrected 21 December 2022)

\begin{abstract}
According to the classical theory of Brownian motion, the mean-squared displacement of diffusing particles evolves linearly with time, whereas the distribution of their displacements is Gaussian. However, recent experiments on mesoscopic particle systems have discovered Brownian yet non-Gaussian regimes where diffusion coexists with an exponential tail in the distribution of displacements. Here we show that, contrary to the present theoretical understanding, the length scale $\lambda$ associated with this exponential distribution does not necessarily scale in a diffusive way. Simulations of Lennard-Jones systems reveal a behavior $\lambda \sim t^{1 / 3}$ in three dimensions and $\lambda \sim t^{1 / 2}$ in two dimensions. We propose a scaling theory based on the idea of hopping motion to explain this result. In contrast, simulations of a tetrahedral gelling system, where particles interact by a nonisotropic potential, yield a temperature-dependent scaling of $\lambda$. We interpret this behavior in terms of an intermittent hopping motion. Our findings link the Brownian yet non-Gaussian phenomenon with generic features of glassy dynamics and open new experimental perspectives on the class of molecular and supramolecular systems whose dynamics is ruled by rare events.

DOI: $10.1103 /$ PhysRevX.11.031002

Subject Areas: Condensed Matter Physics

Materials Science, Soft Matter
\end{abstract}

\section{INTRODUCTION}

In one of his celebrated 1905 papers, Einstein proposed a statistical interpretation of Brown's observation based on the corpuscular constitution of matter [1,2]. Einstein's theory predicted two concomitant properties for the probability density function (PDF) of displacements of the Brownian particles: Its shape must be Gaussian and its variance, the mean-squared displacement (MSD), must grow linearly (diffusively) with time. Since the seminal experiments conducted by Perrin more than 100 years ago [3], these two predictions were routinely validated and the coexistence between Gaussianity and diffusivity became a paradigm. Exceptions to this long-standing paradigm were first observed in the realm of anomalous diffusion [4-8], where nonlinear time dependences of the MSD

\footnotetext{
*sandalo@ugr.es
}

Published by the American Physical Society under the terms of the Creative Commons Attribution 4.0 International license. Further distribution of this work must maintain attribution to the author(s) and the published article's title, journal citation, and DOI. Open access publication funded by the Max Planck Society. coexist with both Gaussian and non-Gaussian PDFs of displacements $[4,5]$.

Recent experiments have found a new class of counterexamples to this paradigm. Several mesoscopic particle systems present a time regime where linear diffusion coexists with a non-Gaussian PDF of displacements characterized by an exponential tail $e^{-r / \lambda(t)}$ as a function of the displacement $r$ [9-15]. The exponential tail is controlled by a time-dependent length scale $\lambda(t)$, which evolves as a power law: $\lambda(t) \sim t^{\beta}$ with $\beta>0$. This Brownian yet nonGaussian regime appears in a variety of systems, including colloidal beads moving on the top of lipid tubes $[9,10]$, nanospheres in entangled protein suspensions [9], binary mixtures of colloidal hard spheres [11], microspheres in biological hydrogels [12], and passive tracers in suspensions of eukaryotic swimmers [13]. Some of these works report values for $\beta$ compatible with $1 / 2$ [9-11,13] that have motivated theoretical models based on the idea of diffusing diffusivities [16-20]. These models assume a heterogeneous dynamics: Particles move according to a timedependent diffusion coefficient which leads to a Brownian yet non-Gaussian regime with exponential asymptotics [16-18]. In these models, the variance of the exponential $\lambda^{2}(t)$ is responsible for the total MSD: $\lambda^{2}(t) \sim \operatorname{MSD}(t) \sim t$. 
However, more recent experiments have measured a value of $\beta$ significantly smaller than $1 / 2$ [12], challenging an explanation based on diffusing diffusivities. Far from being an exotic phenomenon with an appealing historical background, this problem has deep implications in the understanding of a broad class of systems which are driven by rare events [10].

Here we study by computer simulations the equilibrium dynamics of representative models of glass formers with isotropic and nonisotropic interactions [21,22]. Our main result is that the length scale $\lambda$ associated with the Brownian yet non-Gaussian regime scales in a nonuniversal way. For systems where particles interact by an isotropic potential, the exponent controlling the evolution of the exponential tail is $\beta=1 / d$, where $d$ is the system dimension. We quantitatively explain this result by a scaling argument based on the idea of hopping motion [22,23]. In glass formers where particles interact by a nonisotropic potential, the exponent $\beta$ also depends on the temperature. This dependence becomes more obvious when the system, which behaves as a strong glass former [21], enters into the Arrhenius regime. We interpret this result as the consequence of an intermittent particle-hopping motion at low temperatures. Finally, we show that the dynamics in the Brownian yet non-Gaussian regime for all the explored systems is the result of mixing the anomalous diffusion of individual particles.

\section{EXPONENTIAL TAIL AND SYSTEM DIMENSION}

We study by molecular dynamics simulations the equilibrium dynamics of isotropic Lennard-Jones particles in two [24] and three dimensions by the Kob-Andersen binary mixture [25] (see Sec. VI). In both cases, the binary nature of the interaction potential is designed to result in a glassy system and, therefore, avoid crystallization even at a very low temperature. In particular, this system shows the super-Arrhenius behavior characteristic of fragile glass formers $[21,24]$. This model also shares many fundamental dynamic and structural properties with other representative systems with isotropic interaction. For instance, its relaxation dynamic mechanism (including the emergence of dynamic heterogeneities) and structural patterns are similar to those observed in other molecular liquids [25,26], colloidal hard spheres [27-29], models of soft spheres [30], and even granular materials [23]. We cover for this system a wide range of temperatures, from the liquid state (slightly above the system onset temperature) to $1.03 T_{c}$, where $T_{c}$ is the estimated system mode coupling temperature (see Sec. IV).

We first measure the particle MSD; see Figs. 1(a) and 1(b). For all temperatures, the MSD presents a characteristic short-time local ballistic motion $\left(\sim t^{2}\right)$ and a long-time diffusive regime $(\sim t)$. Upon cooling, the MSD develops a plateau at intermediate times resulting from an increasingly
Lennard-Jones 3D

Lennard-Jones 2D
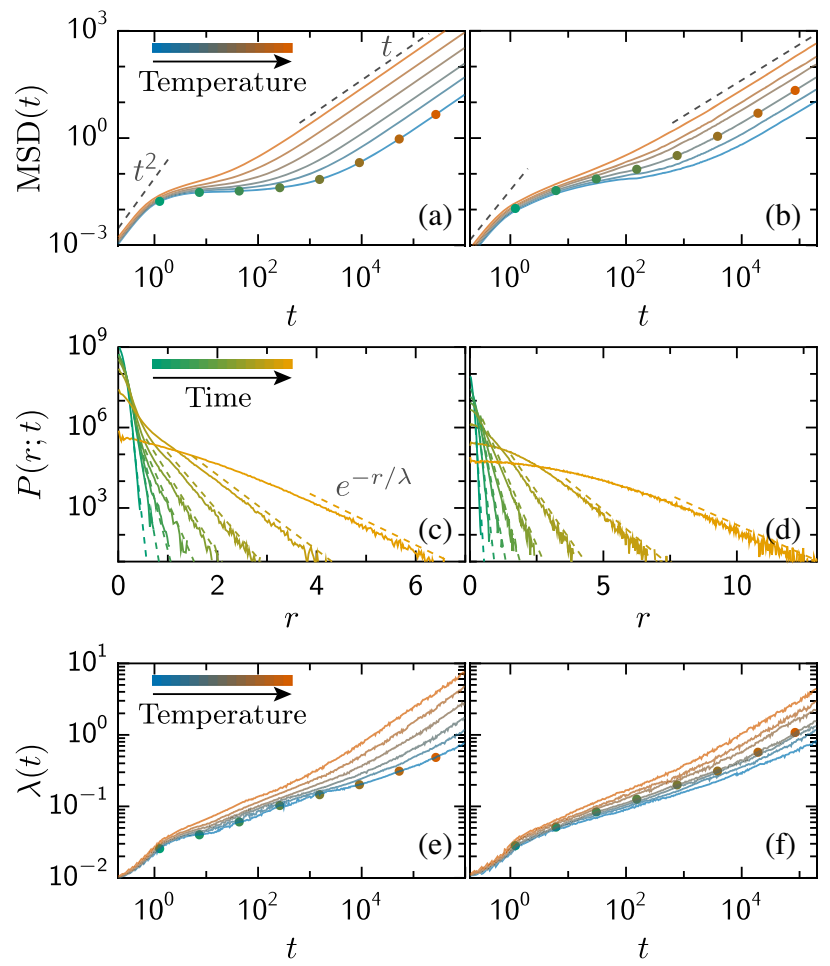

FIG. 1. MSD and exponential tails for the Kob-Andersen binary mixture. (a) MSD for the 3D system at different temperatures from the liquid state to the deep supercooled regime: $T=0.7$, $0.6,0.54,0.50,0.475$, and 0.45 . (b) MSD for the 2D system at different temperatures covering a similar range as in (a): $T=0.6$, $0.50,0.45,0.40,0.36$, and 0.34 . The unit of length is equal to the particle diameter (see Sec. VI for the definition of the time and temperature units). Normalized self-part of the van Hove function $P(r ; t)$ for the 3D system at $T=0.45$ (c) and for the 2D system at $T=0.40(\mathrm{~d})$, where the selected times are marked by dots in the corresponding MSD in (a) and (b). Dashed lines in (c) and (d) show the extent of the exponential range. Characteristic length $\lambda$ as a function of time for the 3D (e) and 2D (f) systems: In both cases, temperatures are as in (a) and (b), respectively. Again, dots signal those times corresponding to $P(r ; t)$ in $(\mathrm{c})$ and $(\mathrm{d})$.

long local residence time, a common feature of all glassforming liquids [22]. We characterize the ensemble distribution of displacements by means of the self-part of the van Hove function [31]

$$
G_{s}(\vec{r} ; t)=\frac{1}{N}\left\langle\sum_{i=1}^{N} \delta\left[\vec{r}-\Delta \vec{r}_{i}(t)\right]\right\rangle
$$

where $G_{s}(\vec{r} ; t)$ is the fraction of particles (from a total number $N)$ which are displaced by $\Delta \vec{r}_{i}(t)=\vec{r}_{i}(t)-\vec{r}_{i}(0)=\vec{r}$ in a time $t$. Since both systems are isotropic, we explore the distribution of displacements as a function of the radial coordinate $r=|\vec{r}|$ and define

$$
P(r ; t) \equiv W(r ; t) / r^{d-1}=\phi_{d} G_{s}(r ; t) ; \quad d \in\{2,3\},
$$


where $W(r ; t)$ is the PDF to have a radial displacement $r, d$ the system dimension, and $\phi_{d}$ the Jacobian angular prefactor (e.g., $\phi_{2}=2 \pi$ and $\phi_{3}=4 \pi$ ) [31]. With this definition, we count the fraction of particles which have displaced radially by $r$ normalized (up to prefactors) by $r^{d-1}$ to account for the volume of the shell within the range $r$ to $r+d r$. In this context, a purely Gaussian diffusion would result in $P(r ; t) \sim e^{-r^{2} / 4 \mathrm{D}(T) t}$, being $D(T)$ the temperature-dependent diffusion coefficient.

The distribution $P(r ; t)$ presents a Gaussian behavior at short distances and an exponential decay at large distances: $P(r ; t) \sim e^{-r / \lambda(t)}$, where $\lambda(t)$ is a characteristic length that increases with time [Figs. 1(c) and 1(d)]. We observe this exponential decay both at low [3D, Fig. 1(c)] and intermediate temperatures [2D, Fig. 1(d)]. Qualitatively similar behaviors to those presented in Figs. 1(c) and 1(d) appear at all the explored temperatures. This observation is compatible with previous works on glassy systems [23] predicting exponential decays (with logarithmic corrections at large $r$ ) for the PDFs of displacements.

To discriminate the $r$ ranges corresponding to the Gaussian and exponential regimes of $P(r ; t)$ and, therefore, accurately estimate $\lambda(t)$, we implement a nonparametric Kolmogorov-Smirnov test (see Sec. VI). The distinction between the two regimes is more evident at a low temperature (in particular, at short and intermediate times), being the two regimes separated by an inflection point in $P(r ; t)$. At longer times, the Gaussian range extends up to larger distance and therefore becomes dominant. In turn, the range of the exponential tail shrinks and thus becomes marginal at long times. Eventually, this takeover leads to a purely Gaussian distribution of displacements as prescribed by the central limit theorem (CLT).

As for the MSD, the growth of $\lambda$ with time is characterized by three distinct regimes [Figs. 1(e) and 1(f)]. At short times, when the MSD is ballistic, $\lambda(t)$ rapidly increases. Displacements within this short time are typically smaller than the particle diameter [Figs. 1(c) and 1(d)] and, therefore, reflect the particle local heterogeneous dynamics. At intermediate times, when the MSD manifests a plateau, $\lambda(t)$ increases in a slower way. In this regime, only a small fraction of particles has abandoned their initial local cage and are able to jump over distances on the order of a few particle diameters [Figs. 1(c) and 1(d)]. At long times, $\lambda(t)$ increases steeply again. In the same time regime, the MSD is compatible with diffusive behavior [Figs. 1(a) and 1(b)]. In the following, we focus on this Brownian yet non-Gaussian regime [10].

Our first goal is to explore the scaling of $\lambda(t)$ within the Brownian yet non-Gaussian regime. We define the time $t_{0}$ at which the 2D and 3D Lennard-Jones systems start to be diffusive $\left[\operatorname{MSD}\left(t \geq t_{0}\right) \sim t\right]$. In practice, we define $t_{0}$ as the time at which the exponent $\mu$ characterizing the scaling of the MSD with time equal to 1 up to a tolerance of 5\%; see Sec. VI for details. We compare the scaling with time of

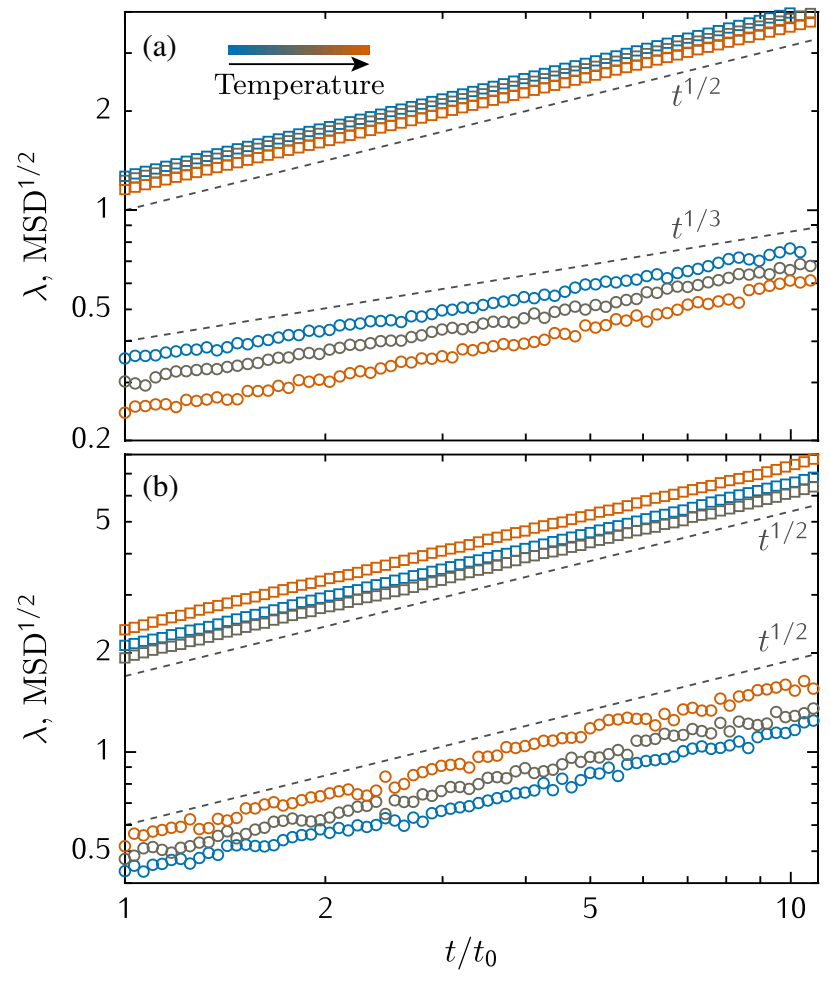

FIG. 2. Brownian yet non-Gaussian regime for the KobAndersen binary mixture. Double-log plot of $\mathrm{MSD}^{1 / 2}$ (squares) and $\lambda$ (circles) as a function of time for the 3D (a) and 2D (b) KobAndersen systems at different temperatures: $T=0.45,0.5$, and 0.6 (3D), and $T=0.4,0.5$, and 0.6 (2D). The $\lambda$ values for the temperature $T=0.4$ for the $2 \mathrm{D}$ system [blue empty circles in (b)] are rescaled by a factor 0.8 to enhance the readability of the figure. Here, $t_{0}$ is the temperature- and system- dependent time at which each system reaches a $\mu$ value compatible with 1 within numerical uncertainty, i.e., $\operatorname{MSD}\left(t \geq t_{0}\right) \sim t^{\mu \cong 1}$. To determine $t_{0}$, we numerically compute the local exponent $\mu(t)$ controlling the time evolution of the $\operatorname{MSD}(t)$, i.e., $\operatorname{MSD}(t) \sim t^{\mu(t)}$ (see Sec. VI). Dashed lines in (a) and (b) serve as a reference.

$\operatorname{MSD}(t)^{1 / 2}$ and $\lambda(t)$ in the time regime $t \geq t_{0}$ for different temperatures (Fig. 2). In both systems, the scaling of the MSD is diffusive, $\operatorname{MSD}^{1 / 2}\left(t \geq t_{0}\right) \sim t^{1 / 2}$. In contrast, the scaling of $\lambda(t)$ is dimension dependent:

$$
\lambda\left(t \geq t_{0}\right) \sim t^{\beta(d)}
$$

The exponent $\beta$ does not appreciably depend on the temperature within the diffusive time window $t \geq t_{0}$. However, $\beta$ clearly depends on the system dimension, with a value compatible with $\beta=1 / 2$ in two dimensions and $\beta=1 / 3$ in three dimensions. While the value $\beta=1 / 2$ in two dimensions is consistent with the diffusive scaling of the MSD, the scaling of $\lambda$ in three dimensions does not seem to be trivially related to that of the MSD. The fact that the scaling of $\lambda$ and the MSD are not, in general, trivially linked descends from the fact that the weight of the exponential tail compared to the rest of the distribution 
is time dependent. While $\lambda$ is a property of the exponential tail only, the MSD is determined by the entire distribution. In this respect, the relative contribution of the exponential tail to the MSD diminishes, with the start of the exponential range moving to larger and larger displacements, as time increases. The scaling of $\lambda$ also contrasts with the interpretation of the Brownian yet non-Gaussian regime based on diffusing diffusivities $[10,16,18]$, where $\lambda^{2}(t) \sim \mathrm{MSD}$ $(t) \sim t$. However, the time evolution we observe for $\lambda\left(t \geq t_{0}\right)$ in three dimensions agrees with recent experiments on microspheres diffusing in biological gels [12], where $\beta<1 / 2$ and the total PDF of displacements presents a Gaussian core, which grows with time, and a marginal exponential tail, which tends to disappear with time.

These observations suggest a general dependence $\beta(d)=1 / d$, with $d \in\{2,3\}$. We propose a theory for such a scaling behavior as an outcome of hopping motion, a signature of all glass-forming liquids below the onset temperature [22]. The exponential tail of $P(r ; t)$ originates from a minority of particles able to escape from their initial cage and perform a large displacement for times of the order of $t_{0}$. We call these particles hoppers and denote by $N_{h}(t)$ their number at time $t$. Since the system is at equilibrium, we assume that hoppers escape from their cage at a constant rate $\omega$ :

$$
N_{h}(t) \approx \omega N t \sim t .
$$

Equation (4) is valid in a time range where $N_{h}(t) \ll N$, being, therefore, $\omega^{-1} \gg t$. We now use that the functional form of the mass density of hoppers $\rho_{h}(r ; t)$ as a function of the distance at time $t$ coincides with that of $P(r ; t)$ for $t \approx t_{0}$ and is, therefore, exponential:

$$
P(r ; t) \sim \rho_{h}(r ; t)=\rho_{0} e^{-r / \lambda(t)} ; \quad t \approx t_{0} .
$$

We consider $\rho_{0}$ to be independent of time for times of the order of $t_{0}$; i.e., the source of hoppers at $r=0$ remains at constant density for $t \approx t_{0}$ since $N_{h}(t) \ll N$. This assumption is consistent with the behavior of $P(r ; t)$ observed in Figs. 1(c) and 1(d), and also clearly manifested by the $P(r ; t)$ shown in the Appendix $\mathrm{C}$, where all the exponential tails at different times cross at a common value at $r \approx 0$. The number of hoppers at a time $t \approx t_{0}$ therefore scales as

$$
\begin{aligned}
N_{h}(t) & \sim \int_{0}^{\infty} r^{d-1} \rho_{h}(r ; t) d r \\
& \sim \int_{0}^{\infty} r^{d-1} e^{-r / \lambda(t)} d r \sim \lambda^{d}(t) .
\end{aligned}
$$

Combining Eqs. (4) and (6), we finally obtain

$$
\lambda(t) \sim t^{1 / d} .
$$

The scaling argument embodied in Eqs. (4)-(6), and leading to Eq. (7) would, in principle, hold for all $d$. In particular, we also test Eq. (7) in Appendix A for $d=4$ for the Lennard-Jones system investigated, also confirming its validity. Nevertheless, the general extension of our scaling argument needs subsequent investigation. In particular, works based on a mode-coupling-theory formalism predict changes in the dynamics of four-point correlators at higher dimensions whose hypothetical effect on the observables studied here should be investigated [32]. Apart from that, and due to the observed emergence of finite-size effects in 2D simulations [33-35], we test and confirm Eq. (7) for a much more larger 2D Lennard-Jones system in Appendix B, in accord with recent experiments in 2D systems [36].

Summarizing, our argument to explain $\lambda(t) \sim t^{1 / d}$ is based on three assumptions: (1) Hoppers leave their local environment at a fixed hopping rate [Eq. (4)]; (2) hoppers fill the space isotropically [Eq. (6)]; (3) the restriction to a time regime $t \approx t_{0}$ where $N_{h}(t) \ll N$. We test these assumptions individually for $d=2,3$ in Appendix C. Once the majority of the particles have abandoned their initial position $\left(t \gg t_{0}\right)$ and present a statistically equivalent jumping record, the last assumption breaks. At such a long time, the PDF of displacements results from a large number of independent and equally distributed displacements and is, therefore, Gaussian by virtue of the CLT.

\section{EXPONENTIAL TAIL AND NONISOTROPIC INTERACTIONS}

We now investigate the evolution of $\lambda(t)$ when particles interact by a nonisotropic potential. To this purpose, we consider a three-dimensional system of tetravalent patchy particles [37-39]. Such particles have four sticky spots tetrahedrally distributed on their surface which provide a strongly directional interaction with fixed valence. Upon cooling, the dynamics slows down and the system develops an amorphous, highly connected tetrahedral network [40]. This model has already shown its capability for capturing some fundamental dynamic and structural features, such as the emergence of an amorphous tetrahedral order present in different classical systems with nonisotropic interactions such as atomistic models of water, silicon, and silica [37,40-44]. We investigate the equilibrium dynamics of this system, which shows the Arrhenius behavior characteristic of a strong glass former $[21,40]$ at moderate density by Brownian dynamics simulations (see Sec. VI) and explore the emergence of the Brownian yet non-Gaussian regime. Our study covers a wide $T$ range from the liquid state (slightly above the percolation threshold) to the deep Arrhenius regime, where the great majority of the particles are tightly bound to their neighbors [37].

In contrast to the Lennard-Jones systems, the scaling of $\lambda(t)$ clearly depends on $T$ for the tetrahedral gelling system (Fig. 3). While at high temperature $\lambda(t) \sim t^{1 / 3}$, compatible with the 3D Lennard-Jones system, the exponent $\beta$ decreases upon cooling the system below the 


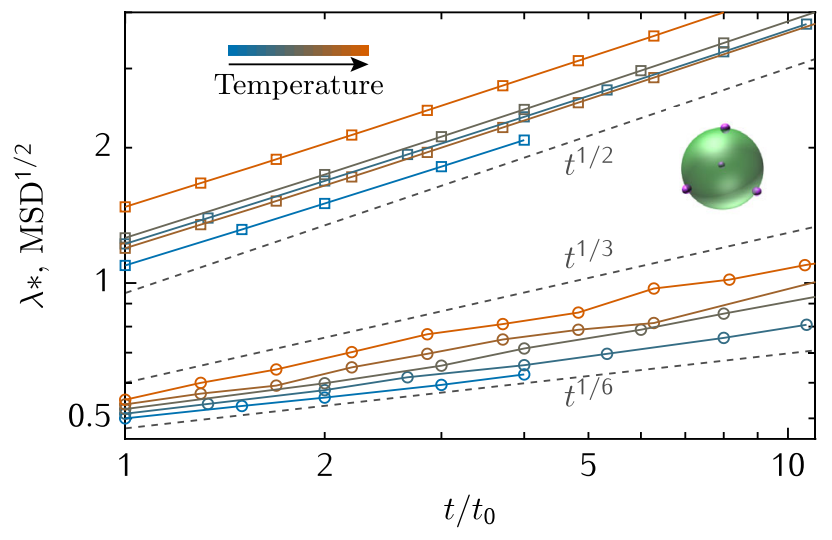

FIG. 3. Brownian yet non-Gaussian regime in the tetrahedral patchy system. Double-log plot of $\mathrm{MSD}^{1 / 2}$ (squares) and $\lambda_{*}$ (circles) as a function of time for the tetrahedral patchy system at different temperatures. To make evident the spread of curves at different temperatures, we present a rescaled value $\lambda_{*}$ for the different temperatures to make them start from an almost common value at $t_{0}$. As in Fig. $2, t_{0}$ is the temperature-dependent time at which the tetrahedral system reaches a value of $\mu$ compatible with 1 . We cover a wide $T$ range from the liquid state to the deep Arrhenius regime: $T=0.1025,0.105,0.11$, 0.115 , and 0.14 (see Sec. VI). The Arrhenius temperature, i.e., the temperature at which the $T$ dependence of the diffusion coefficient becomes exponential, is $T_{A} \approx 0.115[37,40]$ (see also Sec. VI and Fig. 4). Dashed lines serve as a reference. The unit of length is taken as the patchy particle hard-sphere diameter (see Sec. VI). The figure also shows a sketch of a tetrahedral patchy particle.

Arrhenius temperature $[37,40]$, reaching values $\beta \approx 1 / 6$ for temperatures deeply into the Arrhenius regime. Thus, our argument leading to $\beta(d)=1 / d$ independent of $T$ [Eqs. (4)-(7)] does not hold for the gelling system at low $T$. To understand this discrepancy, we individually test the hypotheses underlying our scaling theory (see Appendix C). We find that in this system, Eq. (4) is not satisfied for the gelling system: In particular, the number of hoppers appears to grow sublinearly with time. This observation points to a scenario where the production of hoppers becomes more and more intermittent as the temperature decreases. Such a phenomenon seems to be absent in the Lennard-Jones systems, where our scaling theory holds in all the range of temperatures we explore (see the Appendix). We stress that Eq. (6) is satisfied by our tetrahedral gelling system (see Appendix C) despite the system displaying a fractal structure [40]. This result should, however, be confirmed in other systems showing nontrivial structures $[45,46]$.

We summarize the behavior of the exponent $\beta(T ; d)$ for the three systems (see Fig. 4). For the two Lennard-Jones systems, $\beta$ markedly depends on the system dimension [Eq. (3)] and is practically independent of $T$ (with, at most, a very modest decrease in 3D). Our theoretical argument [Eqs. (4)-(7)] predicts that other systems with isotropic interactions, such as hard and soft spheres, should present

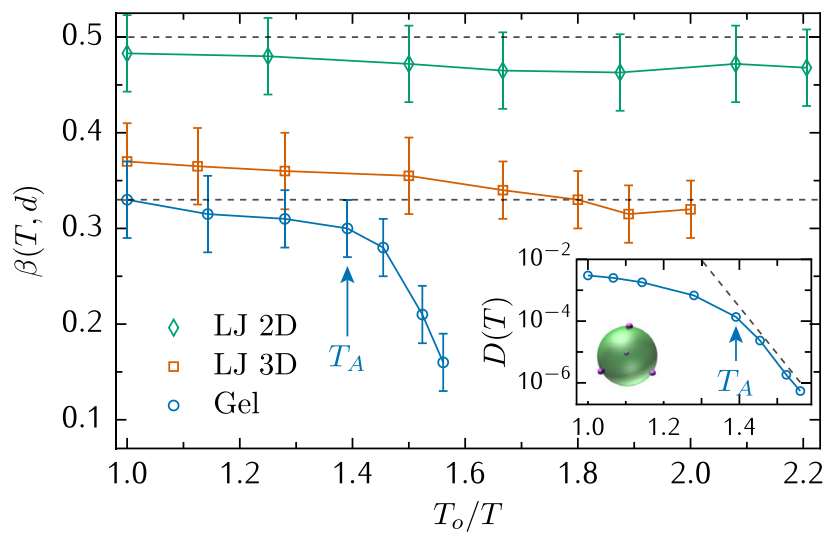

FIG. 4. Compendium of $\beta$ exponents. $\beta(T ; d)$ in the time regime $t \geq t_{0}$ as a function of the temperature for the three systems investigated. Here, $T_{o}$ stands for the onset temperature for the two Lennard-Jones systems and for the percolation temperature for the tetrahedral gelling system (see Sec. VI). The explored $T$ range goes from the liquid state to the deep supercooled regime. Horizontal dashed lines serve as a reference for $1 / 2$ and $1 / 3$. A vertical arrow signals the temperature $T_{A}$ at which the tetrahedral gelling system enters into the Arrhenius regime [40]. Inset: diffusion coefficient $D(T)$ (obtained from the measured MSD) as a function of the temperature for the tetrahedral gelling system (dashed line serves as a reference for the exponential low- $T$ Arrhenius behavior which starts at $T_{A}$ ) [40].

the same behavior. Instead, $\beta$ clearly depends on the temperature for the tetrahedral gelling system. This dependence is stronger once the system enters into the Arrhenius regime, where the diffusion coefficient decreases exponentially upon cooling [40] (inset in Fig. 4), thereby revealing a connection between a defining feature of the dynamics observed in strong glass formers and the emergence of rare events. At higher temperatures, where connectivity is low [37], $\beta$ attains a value compatible with that of the 3D Lennard-Jones system $(\cong 1 / 3)$.

\section{NON-GAUSSIANITY AND DYNAMICS BY POPULATION}

We now look further into the coexistence between linear diffusion and non-Gaussian distributions of displacements in 3D. To this aim, we discriminate the particles into populations according to their potential energy $E$ at $t=0$ as in Ref. [40]. For the tetrahedral gelling system, this procedure leads to five different particle populations characterized by the number of bonds per particle ( $i \in\{0,1,2,3,4\})$ at $t=0$, being $E \mid i$ the potential energy of population $i$ (where $E|i>E| j$ when $i<j$ ) [40]. For the 3D Lennard-Jones system, we arbitrarily define two populations with a marked difference in their initial potential energies: one including the $1 \%$ of particles with the highest potential energy and the other one including the $1 \%$ of particles with the lowest potential energy, both at $t=0$. 

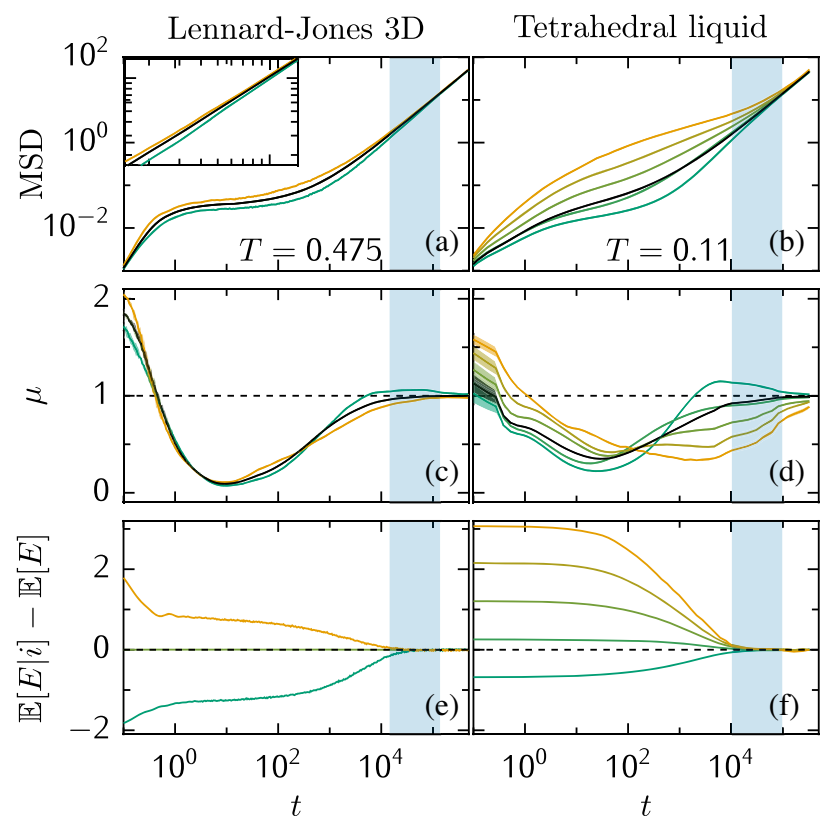

FIG. 5. Dynamics by particle population. Left column corresponds to the 3D Kob-Andersen binary mixture and shows the time evolution of different observables discriminated into two particle populations: (a) $\operatorname{MSD}(t)$, (c) $\mu(t)\left[\operatorname{MSD}(t) \sim t^{\mu(t)}\right]$, and (e) difference between the average potential energy of each population $\mathbb{E}[E \mid i]$, and the system average potential energy $\mathbb{E}[E]$. Yellow (green) lines correspond to the population that includes the $1 \%$ of the particles with the highest (lowest) potential energy at $t=0$. Right column corresponds to the tetrahedral gelling system and shows the same observables as those appearing in the left column [(b), (d), and (f)]. The five colored lines correspond to the five populations having a different number of bonds $(i \in\{0,1,2,3,4\})$ at $t=0$ (lines from yellow to green). Black lines in the whole figure represent observables averaged over all particles. The shaded regions mark out the extent of the Brownian yet non-Gaussian time window, where the exponential tail is still detectable and $\mu(t) \cong$ 1 for the total number of particles. Inset in (a) shows a detail of the MSD corresponding to the shaded time window. The chosen temperatures correspond to the deep supercooled regime (3D Kob-Andersen binary mixture) and the Arrhenius regime (tetrahedral liquid). For both systems, the different populations evolve in such a way that the corresponding observables pass from a short-time behavior (where the particles still retain the information of their initial mechanical state) to a long-time regime, where the particles have lost memory of their initial state and the different populations converge to a common trend.

The time evolution of the MSD for the different populations at fixed $T$ is presented in Figs. 5(a) and 5(b). Populations with high potential energy at $t=0$ show a superdiffusive regime at short times which is compensated with the subdiffusive motion associated with the populations with low potential energy. In particular, for the tetrahedral system, we see that this subdiffusive motion is dominant and represents almost the total MSD. The reason is that, for the chosen temperature, the system is already highly percolated [40], and the populations having a low energy at $t=0$ are much more larger (number of particles) than the populations having a high energy at $t=0$. Thus, while particles with high potential energy are briefly connected to the gel network, low-energy particles are retained for a longer time to the network, resulting in a plausible source for subdiffusion. At intermediate times, the distinct populations reach different values of the MSD at their respective plateaus. This difference is more pronounced for the tetrahedral liquid due to its lower density. When abandoning the plateau, populations with high potential energy at $t=0$ show subdiffusive motion, while populations with low potential energy at $t=0$ present superdiffusive motion. This is particularly clear when looking at the $\mu(t)$ exponent obtained from the slope of the MSD: When leaving the plateau, populations starting with a high potential energy present $\mu(t)<1$, whereas populations starting with a low potential energy show $\mu(t)>1$ [Figs. 5(c) and 5(d)].

The mixing of anomalous diffusions is concomitant with a change in the energy of each population: Particles starting with a high (low) potential energy show a decrease (increase) in their energy which slows down (boosts) their dynamics [Figs. 5(e) and 5(f)]. At long times, once the particles lose the memory of their initial state and pass through all the possible energy states, all the populations show the same average energy while their dynamics converge to a common diffusive trend. It is worth highlighting that this convergence happens at very long times pointing to the existence of a long-term memory of the potential energy. In addition, this convergence shows a common relaxation time which is independent of the particle population. All this phenomenology reduces to a simple intuition: When a particle is in a high- (low-) energy state, it moves faster (slower) than the average. For large times, when all the particles have passed through all the possible fast and slow states, the sampled distributions of displacements for all populations are equivalent, and the total PDF of displacements becomes Gaussian as dictated by the CLT.

Before collapsing into a common diffusive trend, there exists a time window where the different populations still show $\mu(t) \mid i \neq 1 \forall i$, with $\mu(t) \cong 1$ for the total number of particles (shaded region in Fig. 5). This time window corresponds to the Brownian yet non-Gaussian window where the exponential tail is still detectable despite the system as a whole showing $\mu(t) \cong 1$. This observation helps us understand why within this diffusive time window, $P(r ; t)$ is not necessarily Gaussian: The distinct populations have not yet converged and, therefore, their sampled dynamic states are not yet equivalent, resulting in a non-Gaussian PDF of displacements. Our results show that the observed exponential tail originates from mixing anomalies: Within the Brownian yet non-Gaussian regime, each individual population shows its own anomalous diffusion $[\mu(t) \mid i \neq 1]$. 
We further show that, within the Brownian yet non-Gaussian regime, the anomalous diffusion of each population (which is a dynamic feature) can be associated with its initial potential energy (which is a structural feature).

\section{CONCLUSIONS AND PERSPECTIVES}

Brownian yet non-Gaussian transport has recently attracted great interest for its unexpected but ubiquitous presence. It is at the heart of the more general problem of understanding, in a comprehensive way, the rare event dynamics present in many complex systems. Our study brings new insights into this appealing phenomenon. First, we show numerically that for canonical liquid models where particles interact by an isotropic potential, the exponent controlling the evolution of the observed exponential tail is not universal but depends on the system dimension: $\beta(d)=1 / d$. This important finding contrasts with the universality predicted by the current theoretical interpretations. We rationalize this observation by a theoretical scaling argument which does not depend on the specific functional behavior of the interaction potential. For that reason, we expect other representative systems with significantly different potentials, such as hard and soft spheres, to show a similar scaling behavior. Second, we show that dimension is not the only factor affecting the evolution of the exponential tail. In systems where particles interact by a nonisotropic potential, $\beta$ also depends on the temperature. Contrary to the isotropic models investigated in this work (which behave as fragile glass formers), the specific nonisotropic system investigated here behaves as a strong glass former. Therefore, the temperature dependence shown by $\beta$ for the nonisotropic system reveals a new fundamental feature discriminating the dynamics of fragile and strong glass formers, with far-reaching consequences for understanding and classifying glasses. It also establishes a connection between the emergence of the generic Arrhenius regime representative of strong glass formers and their hopping dynamic mechanism. Third, we show that the time regime where the Brownian yet non-Gaussian transport occurs is characterized by a mixed anomalous diffusion of different particle populations. These populations are characterized by a configurational property: their potential energy. We expect our findings for the models investigated here to hold for a large variety of systems. In this respect, the canonical systems we choose in our study share a common fundamental structural and dynamic phenomenology with other representative systems defined by different isotropic and nonisotropic potentials, e.g., hard or soft spheres [27,29,30], and water, silicon, or silica [41-44].

Taken in a broad sense, our results bring new research perspectives, imposing severe constraints to future theories and calling for new experiments. Future theoretical models should account for the dimension and temperature dependences that we observe in this work. For instance, the seminal work by Chaudhuri et al. [23] and the large deviation model proposed by Barkai and Burov [47] predict exponential tails in the PDF of displacements (with logarithmic corrections at large $r$ ) using a continuous-time random-walk (CTRW) formalism. These two approaches do not suggest a dimension-dependent behavior of the characteristic length $\lambda \sim t^{1 / d}$. Other works employing the idea of diffusing diffusivity [16-20,48] lack a dependence on dimension as well. Similar conclusions hold for other approaches to Brownian yet non-Gaussian diffusion, e.g., models mixing CTRW and diffusing diffusivity [49], polymerization models where the fluctuating size of a diffuser generates a diffusing diffusivity process [50,51], diffusion in a fluctuating corrugated channel [52], and colossal Brownian yet non-Gaussian diffusion induced by nonequilibrium noise [53]. Future models should also explain the mixed anomalous diffusion observed in our numerical simulations during the Brownian yet nonGaussian time regime. In our view, this is a crucial yet challenging task. We speculate on the idea of incorporating a CTRW formalism with correlated jumps, one feature that is usually missing in previous works.

From a practical perspective, our results can $a$ priori be tested in several real systems regulated by different control parameters, not necessarily the temperature. For instance, we expect colloidal glass- and gel-forming liquids [22,23] to present a similar phenomenology as the one we describe in this paper when studied as a function of other parameters, e.g., packing fraction. In addition, non-Gaussian tails have also been reported recently in granular systems [54], where shear stress plays the role that temperature plays in the systems we study here. Complex biological media controlled by $p \mathrm{H}$ have also shown Brownian yet nonGaussian transport, where the measured value of $\beta$ is compatible with our results [12]. Finally, we expect a similar phenomenology as the one we report in this work in nonequilibrium active systems, where transport is controlled by an energy of metabolic origin $[55,56]$. In this respect, non-Gaussian regimes have indeed been found in active biological systems constituted by animate energyconsuming particles, for example, in cell migration processes [57] and in the transport of different organelles in the cytoplasm of animal cells [58].

\section{METHODS}

\section{A. Lennard-Jones system}

We perform two- and three-dimensional molecular dynamics simulations of a Kob-Andersen binary mixture [24]. In both cases, we first run simulations in the canonical ensemble to equilibrate the system at a fixed temperature. From them, we run simulations in the microcanonical ensemble to evaluate the dynamic observables presented in this article. The interaction between a 
particle of species $\alpha$ and a particle of species $\beta$ is given by the Lennard-Jones potential

$$
V_{\alpha \beta}(r)=4 \epsilon_{\alpha \beta}\left[\left(\frac{\sigma_{\alpha \beta}}{r}\right)^{12}-\left(\frac{\sigma_{\alpha \beta}}{r}\right)^{6}\right] ; \quad \alpha, \beta \in\{A, B\},
$$

where $A$ and $B$ are the labels for the two species, and $r$ is the distance between the centers of mass of the two particles. For both systems, $\sigma_{A A}=1, \sigma_{A B}=0.8$, $\sigma_{B B}=0.88, \epsilon_{A A}=1, \epsilon_{A B}=1.5$, and $\epsilon_{B B}=0.5$. The potential is truncated and shifted at $r=2.5 \sigma_{\alpha \beta}$ [25]. All the results are given in reduced units, where $\sigma_{A A}$ is the unit of length, $\epsilon_{A A}$ the unit of energy, and $\sigma_{A A} \sqrt{m / 48 \epsilon_{A A}}$ the unit of time (being $m=1$ the mass of the particles). Temperature $T$ is controlled during the equilibration process by an Andersen thermostat with an effective mass of 48 reduced units [24] with Boltzmann's constant set to 1 .

The number of particles for each species of the 2D system is $N_{A}^{2 \mathrm{D}}=6500$ and $N_{B}^{2 \mathrm{D}}=3500$ with a total number density $\rho^{2 \mathrm{D}}=\left(N_{A}^{2 \mathrm{D}}+N_{B}^{2 \mathrm{D}}\right) / L^{2}=1.16$, being $L=92.78$ the length of the square simulation box [24]. For the 3D system, we use a different composition with $N_{A}^{3 \mathrm{D}}=6400$ and $N_{B}^{3 \mathrm{D}}=1600$ for a total number density $\rho^{3 \mathrm{D}}=\left(N_{A}^{3 \mathrm{D}}+N_{B}^{3 \mathrm{D}}\right) / L^{3}=1.20$, being $L=18.80$ the length of the cubic box [25]. These compositions avoid the emergence of a crystal structure even at a very low temperature. We cover temperature ranges $T \in[0.34,0.75]$ (2D) and $T \in[0.45,0.9]$ (3D). For both systems (2D and $3 \mathrm{D})$, the lowest temperature we investigate is $1.03 T_{c}$, where $T_{c}$ is the mode coupling temperature of the glass transition as estimated from data coming from numerical simulations $[24,59]$. The onset temperatures for the $2 \mathrm{D}$ and $3 \mathrm{D}$ systems are $T_{o}^{2 \mathrm{D}}=0.75$ [60] and $T_{o}^{3 \mathrm{D}}=0.9$ [61] (Fig. 4 in the main text). Both systems show at low $T$ the super-Arrhenius dynamic behavior characteristic of fragile glass formers [24]. We use for both systems a velocity Verlet algorithm with a time step depending on the temperature: $\delta t^{2 \mathrm{D}}=0.02$ $\left(\delta t^{2 \mathrm{D}}=0.01\right)$ for $T \leq 0.6(T>0.6)$ and $\delta t^{3 \mathrm{D}}=0.02$ $\left(\delta t^{3 \mathrm{D}}=0.01\right)$ for $T \leq 0.7(T>0.7)$. The runs extend over $10^{7}$ and $5 \times 10^{7}$ time steps for the $2 \mathrm{D}$ and $3 \mathrm{D}$ systems, respectively.

The results shown in this article correspond in both cases to particles of the species $A$. For the 2D system, we average over 50 independent simulations (around 325000 individual particle trajectories) for each value of the temperature running on a high-end CPU processor cluster with a total amount of CPU time of 11.5 years. For the 3D system, we average over 70 independent simulations (around 450000 individual particle trajectories) for each value of the temperature with a total amount of CPU time of 18.5 years.

\section{B. Tetrahedral system}

We perform three-dimensional Brownian dynamics simulations of tetravalent patchy particles in the canonical ensemble. The number of particles is $N=10000$ with a length of the cubic simulation box $L=25.98 \sigma$, being $\sigma$ the particle hard-sphere-like diameter, here taken as the unit of length. The number density is $\rho=N / L^{3}=0.57$. For this density, the system develops a homogeneous amorphous tetrahedral network even at a very low temperature [40,62]. The interaction potential comprises a spherical steep repulsion and a short-range attraction. The interaction between a generic pair of particles 1 and 2 is given by

$$
V(1,2)=V_{\text {c.m. }}(1,2)+V_{P}(1,2),
$$

where $V_{\text {c.m. }}(1,2)$ is the repulsive part of the potential between particles 1 and 2, whereas $V_{P}(1,2)$ is the attractive part of the potential between the patches of particles 1 and 2. These potentials are modeled as follows:

$$
\begin{gathered}
V_{\text {c.m. }}(12)=\left(\frac{\sigma}{r_{12}}\right)^{p} \\
V_{P}(12)=-\sum_{i=1}^{M} \sum_{j=1}^{M} \epsilon \exp \left[-\frac{1}{2}\left(\frac{r_{12}^{i j}}{\alpha}\right)^{q}\right] .
\end{gathered}
$$

Here, $r_{12}$ is the distance between the center of mass of particles 1 and 2, $r_{12}^{i j}$ is the distance between patch $i$ on particle 1 and patch $j$ on particle 2 , and $M=4$ is the number of patches per particle. The four patches are tetrahedrally distributed on the surface of the particles. Exponents in $V_{\text {c.m. }}(1,2)$ and $V_{P}(1,2)$ are taken as $p=200$ and $q=10$ to resemble the functional behaviors of a hard sphere and a square well interaction, respectively. We select $\alpha=0.12$ as the patch diameter to avoid having more than one bond per patch and $\epsilon=1.001$, for which the minimum of the attractive part of the potential energy in a bounded configuration is $u_{0} \equiv \min V_{P}(12)=-1$. Temperature $T$ is measured in units of the potential well, taking Boltzmann's constant as 1 . The unit of time is $\sigma \sqrt{m /\left|u_{0}\right|}$, being $m=1$ the mass of the particles. To integrate the equations of motion, we use a velocity Verlet algorithm with a fixed time step $\delta t=0.001$ using a modified Brownian thermostat which explicitly avoids unphysical decorrelations in the particle velocity $[38,63]$. The longest runs extend over $7 \times 10^{9}$ time steps for the lowest temperatures $(T=0.105$ and $T=0.1025$ ). All simulations are performed using the OXDNA simulation package running on GPUs [64].

We investigate temperatures within the range $T \in$ $[0.1025,0.16]$, covering a slowing-down of the dynamics of 4 orders of magnitude. The dynamics of this system exhibits Arrhenius behavior at low temperature, a signature of strong glass formers [40]. The temperature at which the system enters into the Arrhenius regime is $T_{A} \cong 0.115$ 
[37,40] (Fig. 4 in the main text). We take as the onset temperature for this system $T_{0}=0.16$ (Fig. 4 in the main text). Above this temperature, the system is not completely percolated into an infinite cluster, and the MSD does not present any detectable plateau [37].

We evaluate dynamic observables by an average of 50 independent simulations (500000 individual particle trajectories) per each value of the temperature. The total amount of GPU time is approximately two months, which for this system corresponds to more than three years of CPU time on a high-end processor [64].

\section{Estimation of $\lambda(t)$}

We determine $\lambda(t)$ via maximum likelihood assuming an exponential probability density of displacements from a given threshold $r_{t}$. The likelihood at time $t$ is given by

$$
\mathcal{L}_{t}=\prod_{i=1}^{M_{t}} \exp \left(-\frac{r_{i}-r_{t}}{\lambda_{t}}\right) / \lambda_{t}
$$

where $M_{t}$ is the number of individual particle trajectories with a displacement $r_{i}$ larger than $r_{t}$ at time t. We consider for convenience the minus logarithm of the likelihood

$$
l_{t}=-\log \mathcal{L}_{t}=\sum_{i=1}^{M_{t}} \frac{r_{i}-r_{t}}{\lambda_{t}}+M_{t} \log \lambda_{t} .
$$

We then obtain the $\hat{\lambda}_{t}$ that minimizes $l_{t}$ with respect to $\lambda_{t}$ :

$$
\left.\frac{\partial l_{t}}{\partial \lambda_{t}}\right|_{\lambda_{t}=\hat{\lambda}_{t}}=0 \Rightarrow \hat{\lambda}_{t}=\frac{1}{M_{t}} \sum_{i=1}^{M_{t}}\left(r_{i}-r_{t}\right) .
$$

It is desirable to have the lowest possible threshold $r_{t}$ so that more data are included in the estimation while still having a statistically significant exponential tail. In order to achieve this, we use a standard iterative procedure for power-law tail evaluation [65]. We start with a very large threshold $r_{t}$ and compute $\hat{\lambda}_{t}$ by means of Eq. (14). At this point, we perform a test of statistical significance: If the test is passed, i.e., the data are well represented by the estimated exponential distribution, we decrease the threshold $r_{t}$ by $0.025 \sigma$ (being $\sigma$ the particle diameter) and repeat the procedure; if the test fails, we stop the procedure and take $\lambda(t)=\hat{\lambda}_{t}$.

The statistical test we use is the nonparametric Kolmogorov-Smirnov test [66], which measures how extreme the distance is between the theoretical cumulative distribution under the null hypothesis (where the data are sampled from the theoretical distribution) and the empirical cumulative distribution. The distance between distributions is measured by the Kolmogorov-Smirnov statistics,

$$
\mathrm{KS}_{M_{t}}=\sup _{r_{i}(t)}\left|F_{\text {null }}\left(r_{i}(t)\right)-F_{\text {emp }}\left(r_{i}(t)\right)\right|,
$$

where $F_{\text {null }}(x)$ is the cumulative distribution under the null hypothesis (exponential distribution with exponential rate $\hat{\lambda}_{t}$ ) and $F_{\text {emp }}(x)$ the empirical cumulative distribution. The null hypothesis is accepted (rejected) when the $p$ value is greater (smaller) than 0.05 [66].

\section{Estimation of $\mu(t), t_{0}$, and $\beta$}

We define $y_{t} \equiv \operatorname{MSD}(t)$. Since each $y_{t}$ is obtained as an average over many independent and equivalent simulations (see previous sections), we assume that it is normally distributed. We denote by $\bar{y}_{1, T}$ and $\sigma_{1, T}$ the mean and standard deviation of this distribution for a discrete time interval $\left\{t_{1}, \ldots, t_{T}\right\}$. The corresponding likelihood is

$$
\mathcal{L}_{1, T}=\prod_{t=t_{1}}^{t_{T}} \exp \left(-\frac{\left(y_{t}-\bar{y}_{1, T}\right)^{2}}{2 \sigma_{1, T}^{2}}\right) / \sqrt{2 \pi \sigma_{1, T}^{2}} .
$$

As in the previous section, we consider the minus logarithm of the likelihood

$$
\begin{aligned}
l_{1, T} & =-\log \mathcal{L}_{1, T} \\
& =\sum_{t=t_{1}}^{t_{T}} \frac{\left(y_{t}-\bar{y}_{1, T}\right)^{2}}{2 \sigma_{1, T}^{2}}+\frac{T}{2} \log 2 \pi+T \log \sigma_{1, T} .
\end{aligned}
$$

We then assume that $\bar{y}_{1, T}$ and $\sigma_{1, T}$ scale with the same power-law exponent $\mu_{1, T}$ within the discrete time interval $\left\{t_{1}, \ldots, t_{T}\right\}: \bar{y}_{1, T}=a t^{\mu_{1, T}}$ and $\sigma_{1, T}=c t^{\mu_{1, T}}$, with $a, c \in \mathbb{R}^{+}$. This assumption is valid when deviations from normality are small, where the variance $\sigma_{1, T}^{2}$ scales as $2 y_{t}^{2} /(T-1)$ and, therefore, $\sigma_{1, T} \sim \bar{y}_{1, T}$. This common scaling results in

$$
\begin{aligned}
l_{1, T}= & \sum_{t=t_{1}}^{t_{T}} \frac{\left(y_{t}-a t^{\mu_{1, T}}\right)^{2}}{2 c^{2} t^{2 \mu_{1, T}}} \\
& +\frac{T}{2} \log 2 \pi+T \log c+\mu_{1, T} T \log t .
\end{aligned}
$$

This function is minimized with respect to $\mu_{1, T}, a$, and $c$ via the L-BFGS-B algorithm implemented in SCIPY.OPTIMIZE.MINIMIZE [67] and following the procedure detailed in Ref. [68].

In Eq. (18), $\mu_{1, T}$ corresponds to the discrete window given by $\left\{t_{1}, \ldots, t_{T}\right\}$, which is chosen to cover one decade in time. To associate $\mu_{1, T}$ with a specific time, we choose the middle time in the sequence, i.e., $t_{(1+T) / 2}$, being $T$ an odd natural number. In this way, the value $t_{0}$ associated with the entrance to the diffusive regime is the middle time in that sequence $\left\{t_{1}, \ldots, t_{T}\right\}$ for which $\mu_{1, T}=\mu\left(t_{(1+T) / 2}\right) \equiv$ $\mu\left(t_{0}\right)=0.95$. The reason for this choice is that $\mu=1$ is an asymptotic limit $(t \rightarrow \infty)$ which is approached from below 
but never reached. The criterion $\mu=0.95$ ensures that the exponent $\mu$ is compatible with $\mu=1$ up to our measured standard error, which is equal to 0.05 on average.

Finally, to estimate $\beta$ we consider time windows of one decade starting at $t_{0}$ (see Figs. 2 and 3), with the exception of those simulations where the Brownian yet non-Gaussian regime does not cover one decade in time (see, for instance, $T=0.1025$ in Fig. 3). Then we fit $\lambda\left(t \geq t_{0}\right)$ by a least-squares regression assuming a power law $\lambda\left(t \geq t_{0}\right)=a_{1} t^{\beta}+a_{2}$, with $a_{1}, a_{2} \in \mathbb{R}^{+}$as free parameters.

\section{ACKNOWLEDGMENTS}

We thank Walter Kob for fruitful exchanges about this work and Lorenzo Rovigatti for his assistance with the tetrahedral system simulations. We also thank the anonymous reviewers for their careful reading of our manuscript and their insightful comments and suggestions. A. V. C. acknowledges partial financial support from the Deutsche Forschungsgemeinschaft (DFG Grant No. ME 1535/7-1). S. R.-V. acknowledges support from the European Commission through the Marie Skłodowska-Curie Individual Fellowship No. 840195-ARIADNE.

\section{APPENDIX A: SCALING IN A 4D LENNARD-JONES SYSTEM}

In this Appendix, we provide evidence of the scaling behavior $\lambda(t) \sim t^{1 / d}$ for the Lennard-Jones system in $d=4$ from the analysis of the exponential tails of $P(r ; t)=\phi_{4} G(r ; t)$, where $\phi_{4}=2 \pi^{2}$ [see Eq. (2)]. We perform simulations for a $4 \mathrm{D}$ Kob-Andersen binary mixture with the same interaction parameters and units defined in Sec. VI for $d=2,3$. We simulate a system with $N=10^{4}$ particles (5 times the number of particles used in Ref. [24]), with $N_{A}=6500$ and $N_{B}=3500$. The linear size of the simulation box is $L=8.493581$. The total simulation time is $1.6 \times 10^{5}$, and the total number of independent trajectories of $A$ particles analyzed $1.3 \times 10^{4}$. The composition and number density is the same as in Ref. [24] for $d=4$. We investigate two temperatures using an integration time step $\delta t^{4 \mathrm{D}}=0.02$. The higher $T$ is an intermediate temperature which presents an incipient plateau at intermediate times for the MSD, whereas the lower temperature presents a marked plateau at intermediate times [Fig. 6(a)]. With the chosen temperatures. we enclose a wide range from the beginning of the glassy dynamics to the inner supercooled regime, leaving a more detailed study of the functional behavior of $\beta(T)$ in $4 \mathrm{D}$ to future studies.

As in Fig. 2, we fit in Fig. 6(b) the scaling of $\lambda(t)$ for the 4D Lennard-Jones system within a time window where $t \geq t_{0}$ for the two temperatures investigated. This scaling is compatible with $\lambda(t) \sim t^{\beta}$. The fitted values of $\beta$ are $\beta(T=$ $0.750)=0.29 \pm 0.05$ and $\beta(T=0.675)=0.28 \pm 0.04$.
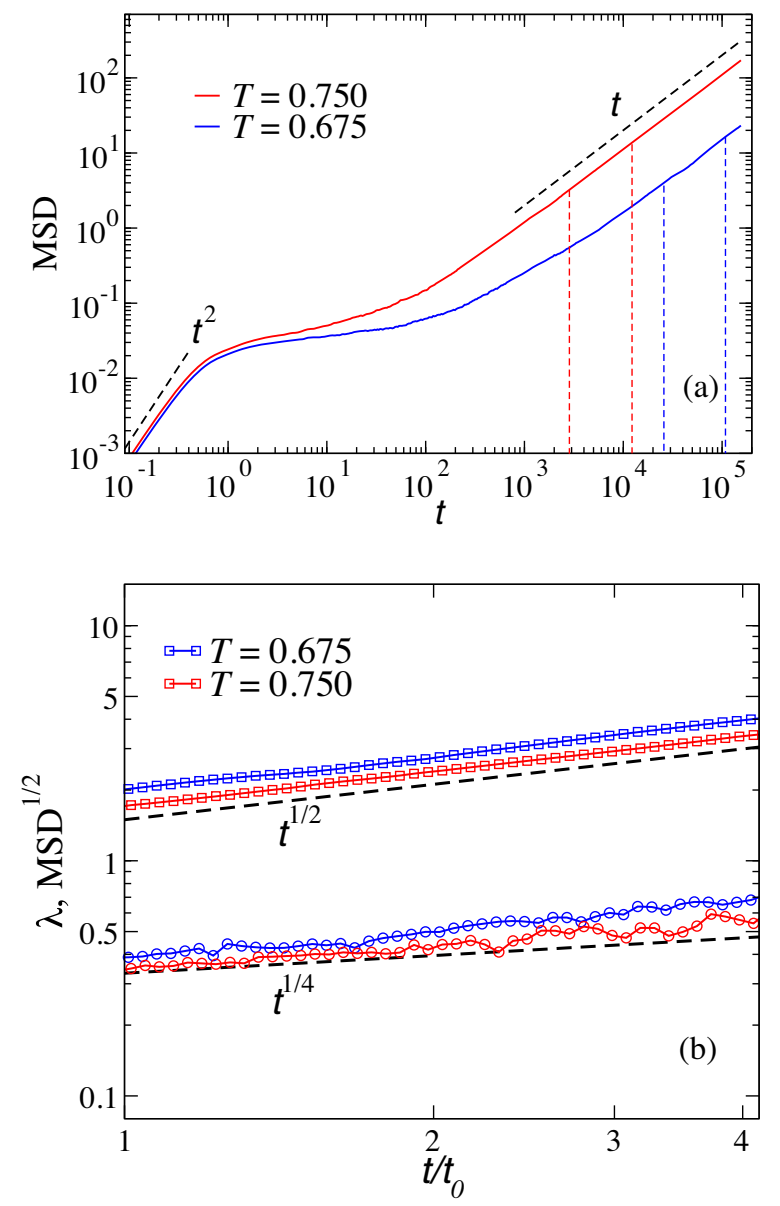

FIG. 6. MSD and Brownian yet non-Gaussian regime for the 4D Kob-Andersen binary mixture. (a) Double-log plot of the MSD for the two temperatures investigated, covering the whole time regime from ballistic to diffusive motion. We mark by vertical dashed lines the time windows corresponding to the analyzed Brownian yet non-Gaussian regime for each temperature. (b) Double-log plot of $\mathrm{MSD}^{1 / 2}$ (squares) and $\lambda$ (circles) as a function of time for the 4D Kob-Andersen binary mixture at different temperatures: $T=0.750$ and 0.675 . Here, $t_{0}$ is the temperature-dependent time at which the system reaches a $\mu$ value compatible with 1 within numerical uncertainty, i.e., $\operatorname{MSD}\left(t \geq t_{0}\right) \sim t^{\mu \cong 1}$ (see Sec. VI). The analyzed time windows correspond to the time regimes signaled by vertical dashed lines in (a). Dashed lines representing power-law behaviors in (a) and (b) serve as a reference.

Both of these values are compatible with the theoretical value $\beta=1 / 4$ as predicted by the argument embodied in Eqs. (4)-(6).

Because of computer limitations, our statistics for the 4D Lennard-Jones system results in a time window smaller than one decade. For $t \gtrsim 4 t_{0}$ the data become noisy, and the exponential tails are in general not accepted by the Kolmogorov-Smirnov test within a sufficiently large $r$ range. To verify the reliability of the results, we perform an additional chi-squared goodness-of-fit test using three 
families of fundamental increasingly monotonic functions, all of them with two free parameters. First, we fit $\lambda(t)$ within the explored time window using the power law predicted by our scaling argument $\alpha t^{\beta}$, with $\alpha>0$ and $\beta>0$ as free parameters. Second, we fit $\lambda(t)$ using the family $\alpha e^{\beta t}$, exactly for the same time window and the same number of points (50 in the plot). Finally, we fit $\lambda(t)$ using the family $\alpha+\beta \log (t)$, again for the same time window and number of points. Thus, for the same number of degrees of freedom, the goodness of the test for the power law is greater than $99 \%$ for the two temperatures investigated, whereas the goodness of the exponential family is less than $30 \%$ and the logarithmic family less than $1 \%$. This statistical test supports the power-law behavior and the value $\beta \cong 1 / 4$ predicted by our scaling argument.

\section{APPENDIX B: FINITE-SIZE EFFECTS IN A 2D LENNARD-JONES SYSTEM}

In this Appendix, we explore whether finite-size effects influence our results for the 2D Lennard-Jones system. We compare the system presented in Sec. VI with an equivalent larger system (same composition, number density, and interaction parameters). We choose an intermediate temperature $T=0.6$ already presented in Fig. 2(b). The larger system has a total number of particles $N=4 \times 10^{4}$, with $N_{A}=2.6 \times 10^{4}$ and $N_{B}=1.4 \times 10^{4}$, i.e., 4 times the number of particles in the system described in Sec. VI. The linear size of the simulation box is $L=185.56246$ (twice the size in the system described in Sec. VI). The total simulation time is $2 \times 10^{4}$, and the total number of independent trajectories of $A$ particles $6 \times 10^{5}$ (the same number of trajectories in the system described in Sec. VI at $T=0.6)$.

In Fig. 7(a), we show a comparison of the MSD for the two systems. We appreciate only a tiny difference in the diffusive regime, where the larger system seems to present a slightly greater diffusion coefficient than the small system. Thus, as shown in Fig. 7(b), while the two systems arrive at the diffusive regime almost at the same time, there is a small shift in both the MSD and $\lambda$ within the Brownian yet non-Gaussian regime. In any case, when comparing the dynamics of the two systems within this regime, we see that both systems present a common power-law behavior compatible with $\beta=1 / 2$, as already documented for the small system in Sec. II. The fitted values are $\beta($ large $)=0.45 \pm 0.05$ and $\beta$ (small $)=$ $0.47 \pm 0.04$. This comparison confirms that finite-size effects negligibly affect our main result for 2D LennardJones systems: the power-law dependence $\lambda(t) \sim t^{1 / 2}$ in the Brownian yet non-Gaussian regime.

We perform an additional comparison for two static observables: potential energy and pressure. The probability densities of the potential energy for the small and large
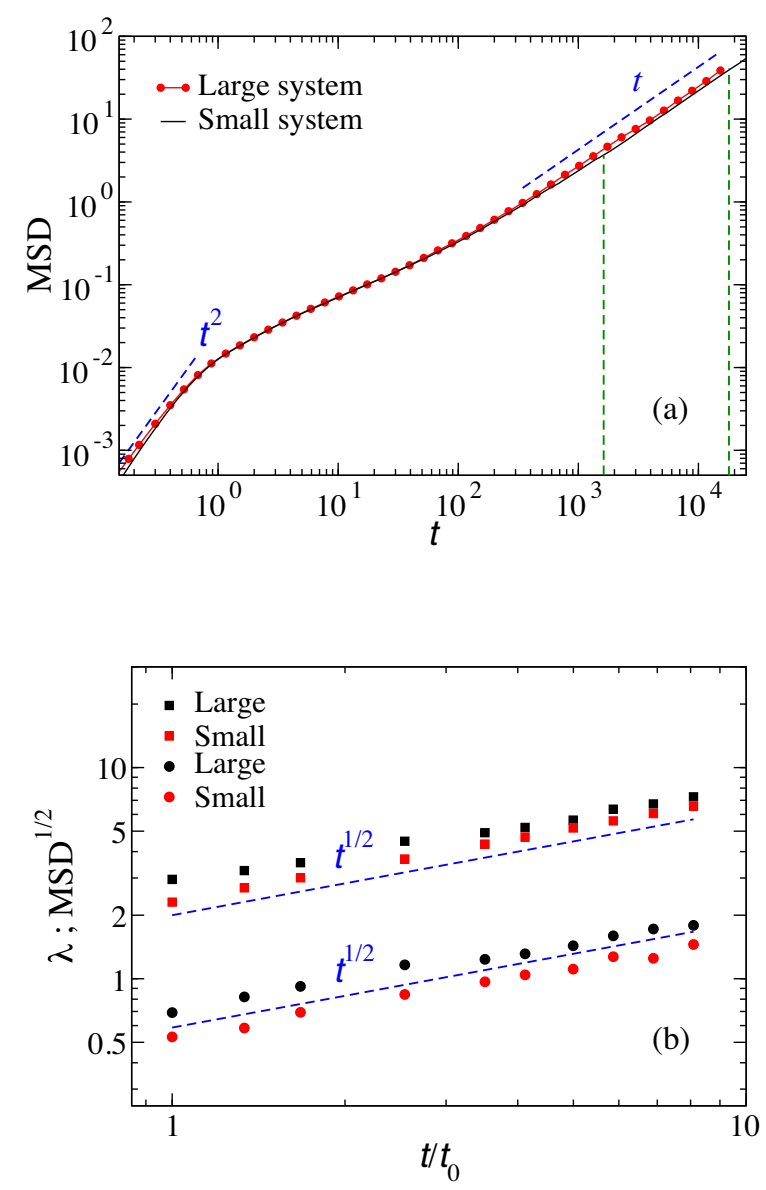

FIG. 7. MSD and Brownian yet non-Gaussian regime for two 2D Kob-Andersen binary mixtures of different size. (a) Double-log plot of the MSD at $T=0.6$ for the large and small system, covering the whole time regime from ballistic to diffusive motion. We mark by vertical dashed lines the time window corresponding to the analyzed Brownian yet non-Gaussian regime. (b) Double-log plot of $\mathrm{MSD}^{1 / 2}$ (squares) and $\lambda$ (circles) as a function of time for the large and small system at $T=0.6$. Here, $t_{0}$ is the temperaturedependent time at which the systems reach a $\mu$ value compatible with 1 within numerical uncertainty, i.e., MSD $\left(t \geq t_{0}\right) \sim t^{\mu \cong 1}$ (see Sec. VI). The analyzed time window corresponds to the time regime signaled by vertical dashed lines in (a). Dashed lines representing power-law behaviors in (a) and (b) serve as a reference.

systems are peaked at about the same mean value: -3.139 for the large system and -3.140 for the small system; see Fig. 8(a). The difference between the two distributions is in their standard deviations: $\delta_{E}($ large $)=0.007$ and $\delta_{E}($ small $)=0.015$. As expected by the law of large numbers, the standard deviation decreases when increasing the number of particles as $\delta_{E}($ small $) / \delta_{E}($ large $) \approx$ $\sqrt{N_{\text {large }} / N_{\text {small }}}=2$. In Fig. $8(\mathrm{~b})$, we show similar results for the pressure: a common mean value of 6.60 for the two systems and a factor 2 in the standard deviations, $\delta_{P}($ large $)=0.06$ and $\delta_{P}($ small $)=0.12$. 

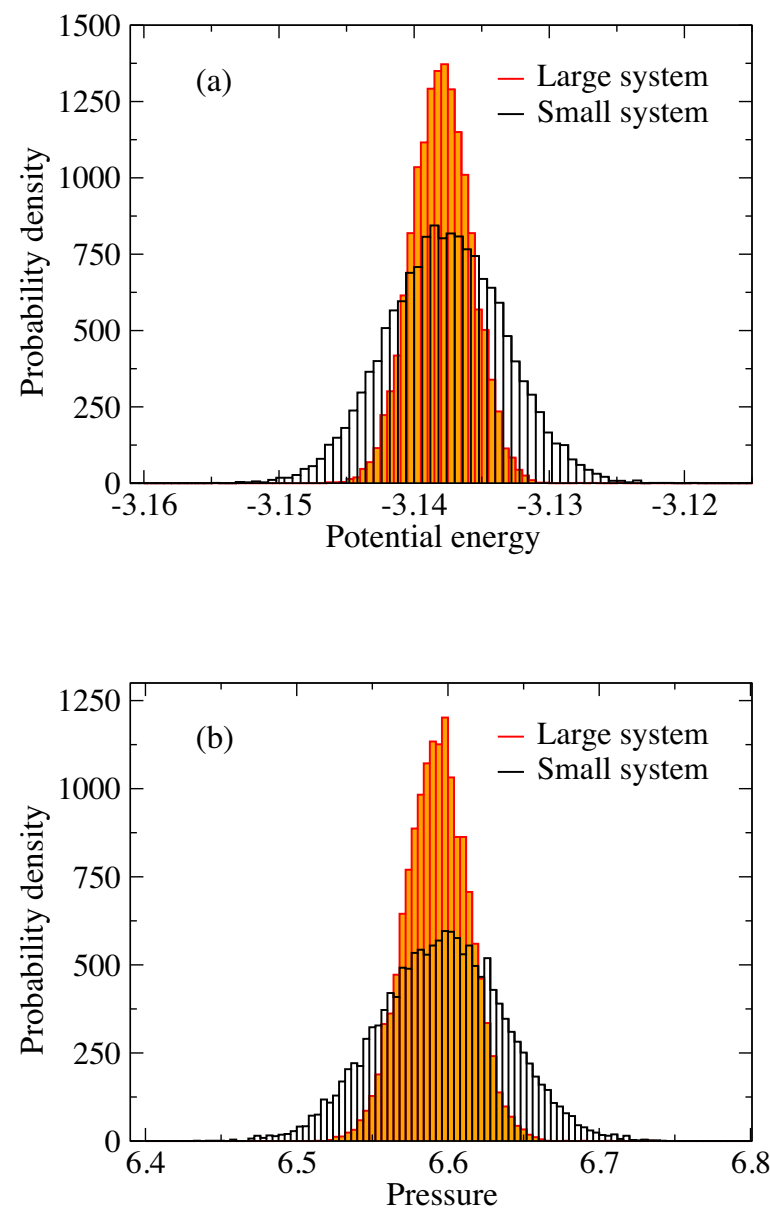

FIG. 8. Potential energy and pressure for two 2D Kob-Andersen binary mixtures of different size. (a) Probability density of the potential energy per particle for the large and small system at $T=0.6$ monitored every 20 time units for a total time $2 \times 10^{4}$. (b) Probability density of pressure for the large and small system at $T=0.6$ monitored as in (a).

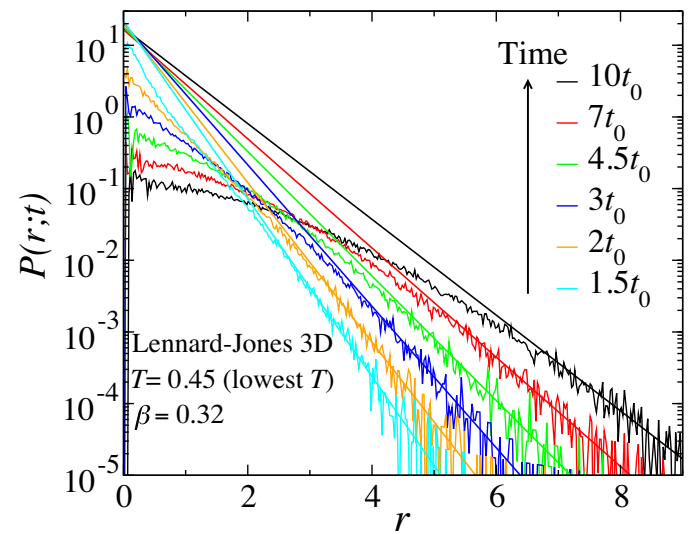

\section{APPENDIX C: TESTING THE SCALING ARGUMENT}

We test the scaling argument embodied in Eqs. (4)-(6) of the main text. We first consider the 3D Lennard-Jones and 3D tetrahedral gel systems at their respective lowest temperatures. We wish in particular to show why the scaling argument fails for the tetrahedral system at low $T$, given as a result a $\beta$ exponent significantly smaller than $1 / 3$ (see Fig. 4 in the main text).

We first focus on $P(r ; t)$ at different times within the diffusive yet non-Gaussian regime $\left(t \geq t_{0}\right)$; see Fig. 9. The different exponential tails extend up to $r=0$ (straight lines in Fig. 9) and all intersect approximately at a common value at $r=0$. Therefore, Eq. (5), which assumes a nonevolving $\rho_{0}$, is well satisfied for both systems. At short distances, $P(r ; t)$ decreases with time for the 3D Lennard-Jones system. In contrast, $P(r ; t)$ for the tetrahedral gelling system presents two favorable distances (inset in Fig. 9, right) at which the probability increases with time, at least within the time regime investigated. One distance corresponds to the average first neighbor distance $(r \cong 1)$ and the other one corresponds to the tetrahedral neighbor distance ( $r \cong 1.7$ ). This result is consistent with the underlying amorphous tetrahedral structure at low $T$ which still manifests itself, despite many particles having already jumped. In other words, some particles have moved to a position at time $t$, which was occupied by another particle at $t=0$.

Our next step is to test Eq. (4) of the main text, which establishes a linear relation between the number of hoppers within the diffusive yet non-Gaussian regime and time: $N_{h}(t) \approx \omega N t \sim t$. We estimate $N_{h}(t)$ by integrating for both systems the exponential tails shown in Fig. 9. This is presented in Figs. 10(a) and 10(b). While the 3D Lennard-Jones system satisfies Eq. (4) for the lowest $T$ investigated $\left[N_{h}(t) \sim t\right]$, the tetrahedral gelling

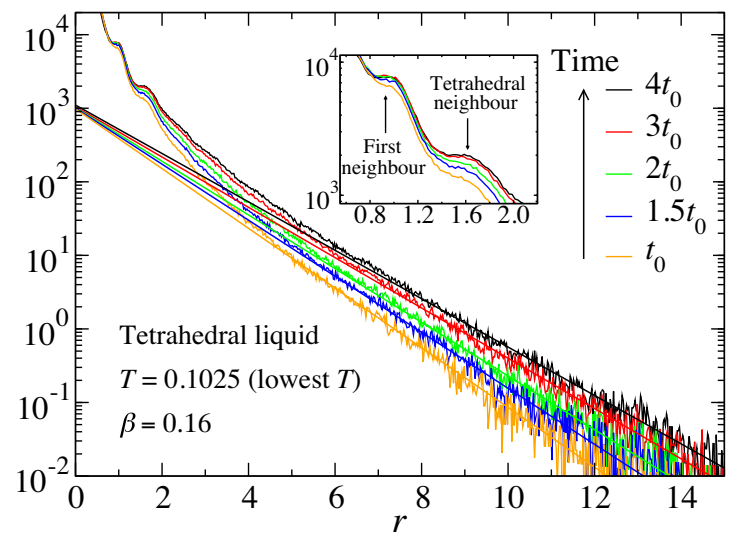

FIG. 9. Left: $P(r ; t)$ at different times $t\left(>t_{0}\right)$ for the 3D Kob-Andersen binary mixture at the lowest temperature investigated $(T=0.45)$. Right: $P(r ; t)$ at different times $t\left(\geq t_{0}\right)$ for the tetrahedral gelling system at the lowest temperature investigated $(T=0.1025)$. The inset in the right figure shows a detail of $P(r ; t)$ at distances corresponding to the first and tetrahedral neighbors. Straight lines for both systems correspond to the exponential tails of the different $P(r ; t)$, extended to $r=0$. 

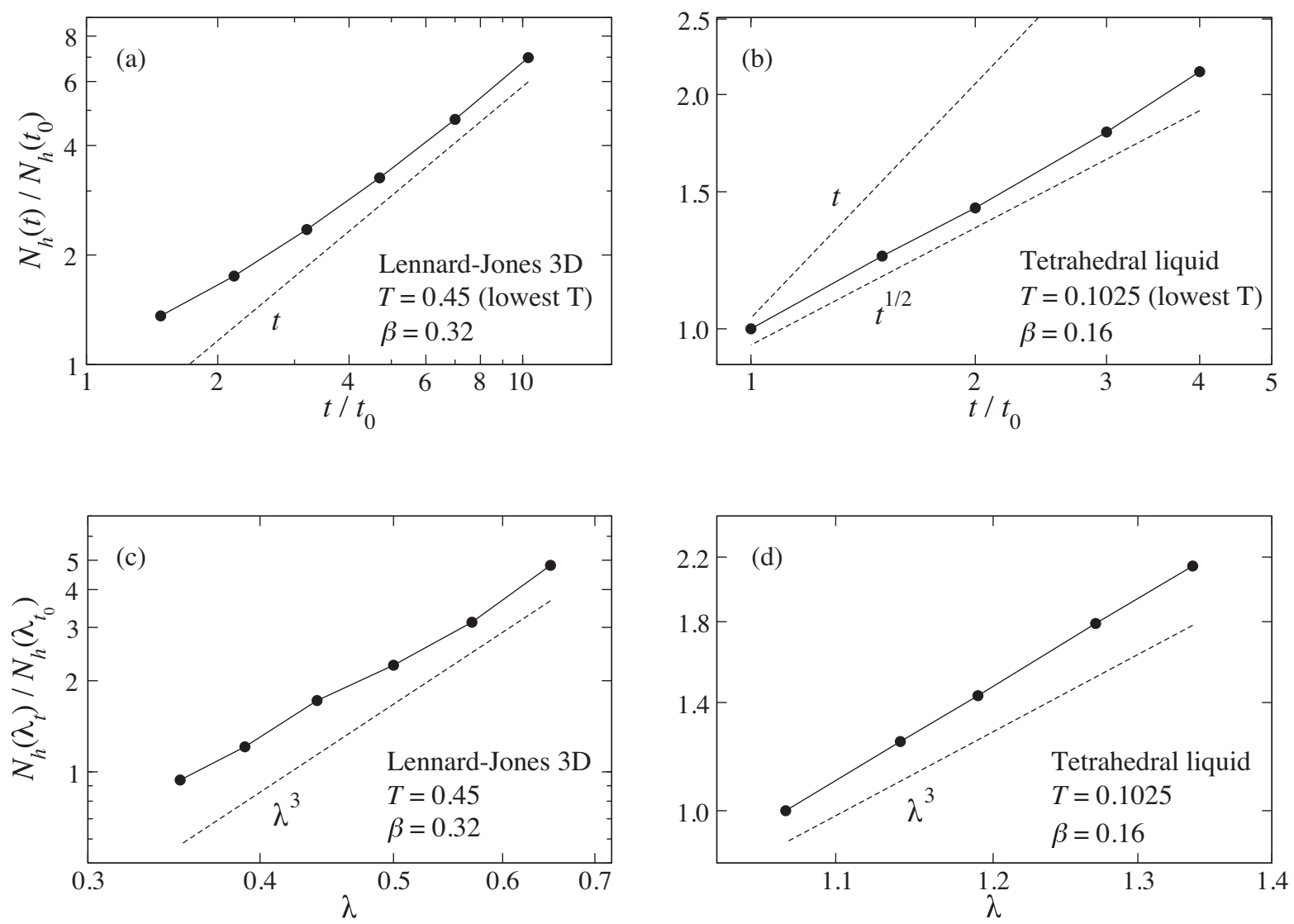

FIG. 10. $N_{h}(t)$ normalized by $N_{h}\left(t_{0}\right)$ for the 3D Kob-Andersen binary mixture at $T=0.45$ (a) and the tetrahedral gelling system at $T=0.1025$ (b) both obtained by integrating the exponential tails shown in Fig. 9. $N_{h}(\lambda)$ normalized by $N_{h}\left(\lambda_{t_{0}}\right)$ for the 3D KobAndersen binary mixture at $T=0.45$ (c) and the tetrahedral gelling system at $T=0.1025$ (d). Dashed lines in all the figures are different power laws shown as references.

system presents a power-law behavior compatible with $N_{h}(t) \sim t^{1 / 2}$, in violation of Eq. (4).

Two different, but nonmutually exclusive, mechanisms could explain why Eq. (4) fails for the tetrahedral system. First, the tetrahedral system maintains a underlying structure at times greater than $t_{0}$. This structure is revealed by the two peaks in the inset of Fig. 9 (right). This means that some particles that have already jumped to a distance corresponding to one of these peaks could in principle jump back to their original positions, thus abandoning the exponential tail. Such a mechanism would slow down the growth of $N_{h}(t)$ with time. Second, we also speculate that the tetrahedral system could produce hoppers intermittently in time. In this way, the tetrahedral system would present avalanches in the production of hoppers alternated with periods of scarce production. As a result, the empirical total production of hoppers given by $N_{h}(t)$ would not be linear before reaching the final Gaussian regime, at which all the particles have already jumped. We leave a detailed test of these hypotheses for future studies.
Finally, we directly test Eq. (6) of the main text by plotting $N_{h}$ versus $\lambda$ in Figs. 10(c) and 10(d). We obtain $N_{h}(\lambda)$ from $N_{h}(t)$ at a fixed time $t$ and take the $\lambda$ value corresponding to this time $t$. In this case, the behavior of both systems is compatible with Eq. (6): $N_{h}(\lambda) \sim \lambda^{d=3}$. In summary, the results of Fig. 10 show $N_{h}(t) \sim t$ and $N_{h}(\lambda) \sim \lambda^{3}$ for the 3D Lennard-Jones system and $N_{h}(t) \sim$ $t^{1 / 2}$ and $N_{h}(\lambda) \sim \lambda^{3}$ for the tetrahedral system. These behaviors result in $\lambda \sim t^{\beta=1 / 3}$ for the 3D Lennard-Jones system and $\lambda \sim t^{\beta=1 / 6}$ for the tetrahedral system (see the $\beta$ exponent at lowest temperature for the 3D Lennard-Jones and the tetrahedral systems in Fig. 4 in the main text).

For completeness, we test our scaling argument in the $2 \mathrm{D}$ Lennard-Jones system at $T=0.36$. The common intersection of the exponential tails is shown in Fig. 11(a), the predicted behavior $N_{h}(t) \sim t$ is presented in Fig. 11(b), and the behavior $N_{h}(\lambda) \sim \lambda^{2}$ in Fig. 11(c). All these partial results lead to $\lambda \sim t^{1 / 2}$, as predicted by the scaling argument [Eqs. (4)-(6)]. 

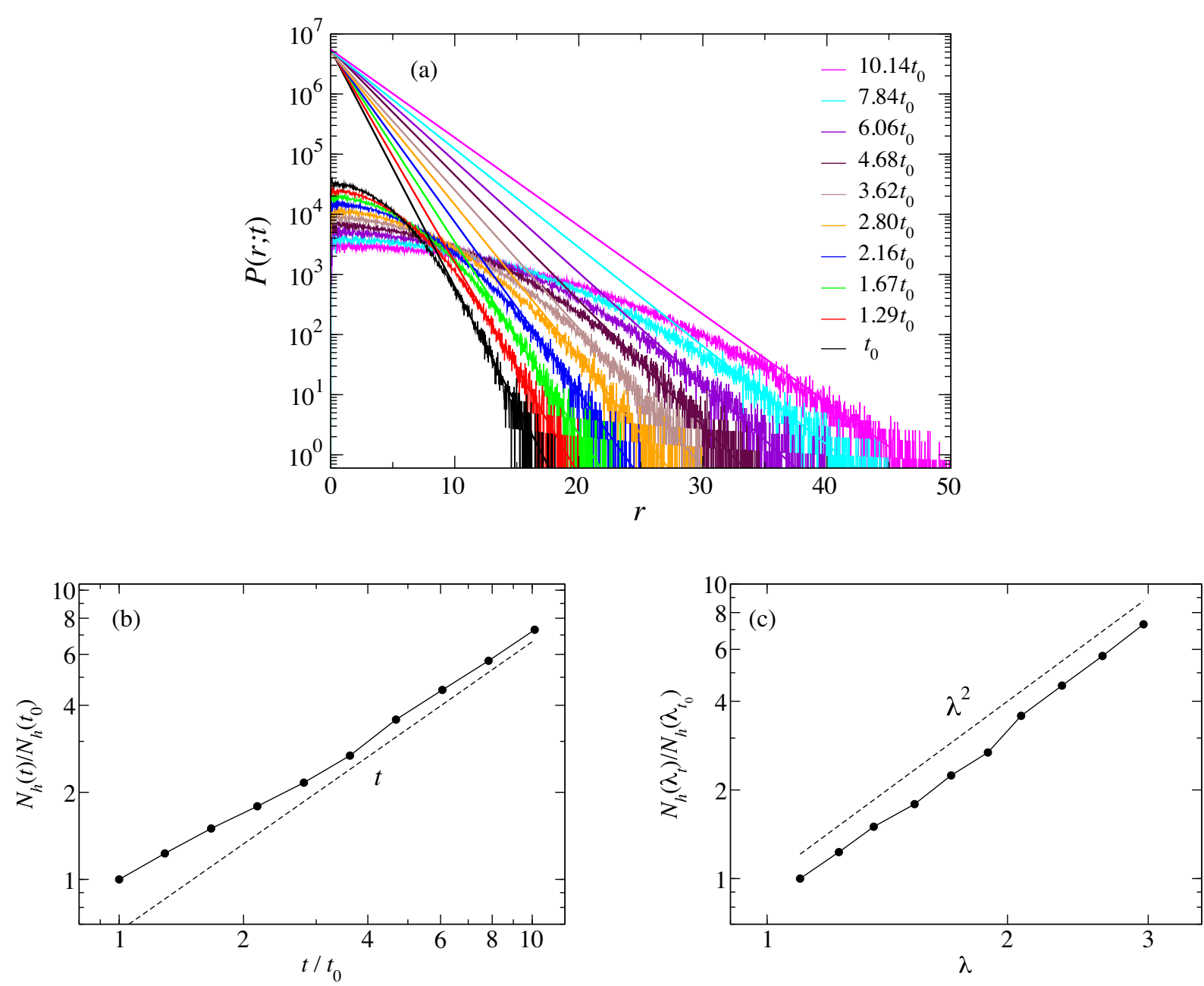

FIG. 11. Testing the scaling behavior for the 2D Kob-Andersen binary mixture at $T=0.36$. (a) $P(r ; t)$ at different times $t$ ( $\left.>t_{0}\right)$, (b) $N_{h}(t)$ normalized by $N_{h}\left(t_{0}\right)$ showing the expected linear behavior in time, (c) $N_{h}(\lambda)$ normalized by $N_{h}\left(\lambda_{t_{0}}\right)$ with the predicted $\lambda^{2}$ behavior.

[1] A. Einstein, Über die von der molekularkinetischen Theorie der Wärme geforderte Bewegung von in ruhenden Flüssigkeiten suspendierten Teilchen, Ann. Phys. (Berlin) 322, 549 (1905).

[2] R. Brown, A Brief Account of Microscopical Observations Made on the Particles Contained in the Pollen of Plants, Philos. Mag. 4, 161 (1828).

[3] J. Perrin, Mouvement brownien et réalité moléculaire, Ann. Chim. Phys. 18, 5 (1909).

[4] J. P. Bouchaud and A. Georges, Anomalous Diffusion in Disordered Media: Statistical Mechanisms, Models and Physical Applications, Phys. Rep. 195, 127 (1990).

[5] R. Metzler and J. Klafter, The Random Walk's Guide to Anomalous Diffusion: A Fractional Dynamics Approach, Phys. Rep. 339, 1 (2000).

[6] R. Klages, G. Radons, and I. E. Sokolov, Anomalous Transport: Foundations and Application (Wiley-VCH, Weinheim, 2008).
[7] I. M. Sokolov, Models of Anomalous Diffusion in Crowded Environments, Soft Matter 8, 9043 (2012).

[8] F. Höfling and T. Franosch, Anomalous Transport in the Crowded World of Biological Cells, Rep. Prog. Phys. 76, 046602 (2013).

[9] B. Wang, S. M. Anthony, S. C. Bae, and S. Granick, Anomalous yet Brownian, Proc. Natl. Acad. Sci. U.S.A. 106, 15160 (2009).

[10] B. Wang, J. Kuo, S.C. Bae, and S. Granick, When Brownian Diffusion Is Not Gaussian, Nat. Mater. 11, 481 (2012).

[11] J. Guan, B. Wang, and S. Granick, Even Hard-Sphere Colloidal Suspensions Display Fickian yet Non-Gaussian Diffusion, ACS Nano 8, 3331 (2014).

[12] C. Wagner, B. Turner, M. Rubinstein, G. McKinley, and K. Ribbeck, A Rheological Study of the Association and Dynamics of MUC5AC Gels, Biomacromolecules 18, 3654 (2017).

[13] K. C. Leptos, J. S. Guasto, J. P. Gollub, A. I. Pesci, and R. E. Goldstein, Dynamics of Enhanced Tracer Diffusion in Suspensions of Swimming Eukaryotic Microorganisms, Phys. Rev. Lett. 103, 198103 (2009). 
[14] M. Matse, M. V. Chubynsky, and J. Bechhoefer, Test of the Diffusing-Diffusivity Mechanism Using Near-Wall Colloidal Dynamics, Phys. Rev. E 96, 042604 (2017).

[15] R. Metzler, Gaussianity Fair: The Riddle of Anomalous yet Non-Gaussian Diffusion, Biophys. J. 112, 413 (2017).

[16] M. V. Chubynsky and G. W. Slater, Diffusing Diffusivity: A Model for Anomalous, yet Brownian, Diffusion, Phys. Rev. Lett. 113, 098302 (2014).

[17] R. Jain and K. L. Sebastian, Diffusion in a Crowded, Rearranging Environment, J. Phys. Chem. B 120, 3988 (2016).

[18] A. V. Chechkin, F. Seno, R. Metzler, and I. M. Sokolov, Brownian yet Non-Gaussian Diffusion: From Superstatistics to Subordination of Diffusing Diffusivities, Phys. Rev. X 7, 021002 (2017).

[19] N. Tyagi and B. Cherayil, Non-Gaussian Brownian Diffusion in Dynamically Disordered Thermal Environment, J. Phys. Chem. B 121, 7204 (2017).

[20] Y. Lanoiselée, N. Moutal, and D. S. Grebenkov, DiffusionLimited Reactions in Dynamic Heterogeneous Media, Nat. Commun. 9, 4398 (2018).

[21] C. C. A. Angell, P. H. Poole, and J. Shao, Glass-Forming Liquids, Anomalous Liquids, and Polyamorphism in Liquids and Biopolymers, Il Nuovo Cimento D 16, 993 (1994).

[22] L. Berthier and G. Biroli, Theoretical Perspective on the Glass Transition and Amorphous Materials, Rev. Mod. Phys. 83, 587 (2011).

[23] P. Chaudhuri, L. Berthier, and W. Kob, Universal Nature of Particle Displacements Close to Glass and Jamming Transitions, Phys. Rev. Lett. 99, 060604 (2007).

[24] R. Brüning, D. A. St-Onge, S. Patterson, and W. Kob, Glass Transitions in One-, Two-, Three-, and Four-Dimensional Binary Lennard-Jones Systems, J. Phys. Condens. Matter 21, 035117 (2009).

[25] W. Kob and H. C. Andersen, Scaling Behavior in the $\beta$-Relaxation Regime of a Supercooled Lennard-Jones Mixture, Phys. Rev. Lett. 73, 1376 (1994).

[26] T. A. Weber and F. H. Stillinger, Local Order and Structural Transitions in Amorphous Metal-Metalloid Alloys, Phys. Rev. B 31, 1954 (1985).

[27] K. N. Pham, S. U. Egelhaaf, P. N. Pusey, and W. C. K. Poon, Glasses in Hard Spheres with Short-Range Attraction, Phys. Rev. E 69, 011503 (2004).

[28] L. Berthier, Time and Length Scales in Supercooled Liquids, Phys. Rev. E 69, 020201(R) (2004).

[29] K. N. Pham, A. M. Puertas, J. Bergenholtz, S. U. Egelhaaf, A. Moussaïd, P. N. Pusey, A. B. Schofield, M. E. Cates, M. Fuchs, and W. C. K. Poon, Multiple Glassy States in a Simple Model System, Science 296, 104 (2002).

[30] L. Berthier, E. Flenner, H. Jacquin, and G. Szamel, Scaling of the Glassy Dynamics of Soft Repulsive Particles: A ModeCoupling Approach, Phys. Rev. E 81, 031505 (2010).

[31] J. Hansen and I. R. McDonald, Theory of Simple Liquids (Academic Press, New York, 2006).

[32] G. Biroli and J.-P. Bouchaud, Diverging Length Scale and Upper Critical Dimension in the Mode-Coupling Theory of the Glass Transition, Europhys. Lett. 67, 21 (2004).
[33] E. Flenner and G. Szamel, Fundamental Differences between Glassy Dynamics in Two and Three Dimensions, Nat. Commun. 6, 7392 (2015).

[34] B. Illing, S. Fritschi, H. Kaiser, C. L. Klix, G. Maret, and P. Keim, Mermin-Wagner Fluctuations in 2D Amorphous Solids, Proc. Natl. Acad. Sci. U.S.A. 114, 1856 (2017).

[35] Y.-W. Li, C. K. Mishra, Z.-Y. Sun, K. Zhao, T. G. Mason, R. Ganapathy, and M. D. Ciamarra, Long-Wavelength Fluctuations and Anomalous Dynamics in 2-Dimensional Liquids, Proc. Natl. Acad. Sci. U.S.A. 116, 22977 (2019).

[36] R. Pastore, A. Ciarlo, G. Pesce, F. Greco, and A. Sasso, Rapid Fickian yet Non-Gaussian Diffusion after Subdiffusion, Phys. Rev. Lett. 126, 158003 (2021).

[37] L. Rovigatti and F. Sciortino, Self and Collective Correlation Functions in a Gel of Tetrahedral Patchy Particles, Mol. Phys. 109, 2889 (2011).

[38] L. Rovigatti, J. Russo, and F. Romano, How to Simulate Patchy Particles, Eur. Phys. J. E 41, 59 (2018).

[39] Y. Wang, D. Breed, V. Manoharan, L. Feng, A. Hollingsworth, M. Weck, and D. Pine, Colloids with Valence and Specific Directional Bonding, Nature (London) 491, 51 (2012).

[40] S. Roldán-Vargas, L. Rovigatti, and F. Sciortino, Connectivity, Dynamics, and Structure in a Tetrahedral Network Liquid, Soft Matter 13, 514 (2017).

[41] F. Smallenburg, L. Filion, and F. Sciortino, Erasing NoMan's Land by Thermodynamically Stabilizing the LiquidLiquid Transition in Tetrahedral Particles, Nat. Phys. 10, 653 (2014).

[42] F. Perakis, K. Amann-Winkel, F. Lehmkuhler, M. Sprung, D. Mariedahl, J. A. Sellberg, H. Pathaka, A. Spah, F. Cavalca, D. Schlesinger, A. Ricci, A. Jain, B. Massani, F. Aubree, C. J. Benmore, T. Loerting, G. Grubel, L. G. M. Petterssona, and A. Nilsson, Diffusive Dynamics Furing the High-to-Low Density Transition in Amorphous Ice, Proc. Natl. Acad. Sci. U.S.A. 114, 8193 (2017).

[43] A. M. R. de Graff and M. F. Thorpe, The Long-Wavelength Limit of the Structure Factor of Amorphous Silicon and Vitreous Silica, Acta Crystallogr. Sect. A 66, 22 (2010).

[44] J. Horbach and W. Kob, Static and Dynamic Properties of a Viscous Silica Melt, Phys. Rev. B 60, 3169 (1999).

[45] A. Meyer, J. Horbach, W. Kob, F. Kargl, and H. Schober, Channel Formation and Intermediate Range Order in Sodium Silicate Melts and Glasses, Phys. Rev. Lett. 93, 027801 (2004).

[46] M. Spanner, F. Höfling, S. C. Kapfer, K. R. Mecke, G. E. Schröder-Turk, and T. Franosch, Splitting of the Universality Class of Anomalous Transport in Crowded Media, Phys. Rev. Lett. 116, 060601 (2016).

[47] E. Barkai and S. Burov, Packets of Diffusing Particles Exhibit Universal Exponential Tails, Phys. Rev. Lett. 124, 060603 (2020).

[48] V. Sposini, A. V. Chechkin, F. Seno, G. Pagnini, and R. Metzler, Random Diffusivity from Stochastic Equations: Comparison of Two Models for Brownian yet NonGaussian Diffusion, New J. Phys. 20, 043044 (2018).

[49] S. Song, S. J. Park, M. Kim, J. S. Kim, B. J. Sung, S. Lee, J. S. Kim, and J. Sung, Transport Dynamics of Complex Fluids, Proc. Natl. Acad. Sci. U.S.A. 116, 12733 (2019). 
[50] F. Baldovin, E. Orlandini, and F. Seno, Polymerization Induces Non-Gaussian Diffusion, Front. Phys. 7, 00124 (2019).

[51] M. Hidalgo-Soria and E. Barkai, Hitchhiker Model for Laplace Diffusion Processes, Phys. Rev. E 102, 012109 (2020).

[52] Y. Li, F. Marchesoni, D. Debnath, and P. K. Ghosh, NonGaussian Normal Diffusion in a Fluctuating Corrugated Channel, Phys. Rev. Research 1, 033003 (2019).

[53] K. Bialas, J. Luczka, P. Hänggi, and J. K. Spiechowicz, Colossal Brownian yet Non-Gaussian Diffusion Induced by Nonequilibrium Noise, Phys. Rev. E 102, 042121 (2020).

[54] B. Kou, Y. Cao, J. Li, C. Xia, Z. Li, H. Dong, A. Zhang, J. Zhang, W. Kob, and Y. Wang, Granular Materials Flow Like Complex Fluids, Nature (London) 551, 360 (2017).

[55] S. Ramaswamy, The Mechanics and Statistics of Active Matter, Annu. Rev. Condens. Matter Phys. 1, 323 (2010).

[56] M. C. Marchetti, J. F. Joanny, S. Ramaswamy, T. B. Liverpool, J. Prost, M. Rao, and R. A. Simha, Hydrodynamics of Soft Active Matter, Rev. Mod. Phys. 85, 1143 (2013).

[57] T. E. Angelini, E. Hannezo, X. Trepat, M. Marquez, J. J. Fredberg, and D. A. Weitz, Glass-like Dynamics of Collective Cell Migration, Proc. Natl. Acad. Sci. U.S.A. 108, 4714 (2011).

[58] B. R. Parry, I. V. Surovtsev, M. T. Cabeen, C. S. O’Hern, E. R. Dufresne, and C. Jacobs-Wagner, The Bacterial Cytoplasm Has Glass-like Properties and Is Fluidized by Metabolic Activity, Cell 156, 183 (2014).

[59] W. Kob and H. C. Andersen, Testing Mode-Coupling Theory for a Supercooled Binary Lennard-Jones Mixture: The van Hove Correlation Function, Phys. Rev. E 51, 4626 (1995).
[60] E. Flenner and G. Szamel, Viscoelastic Shear Stress Relaxation in Two-Dimensional Glass-Forming Liquids, Proc. Natl. Acad. Sci. U.S.A. 116, 2015 (2019).

[61] D. Coslovich and G. Pastore, Understanding Fragility in Supercooled Lennard-Jones Mixtures. I. Locally Preferred Structures, J. Chem. Phys. 127, 124504 (2007).

[62] C. De Michele, S. Gabrielli, P. Tartaglia, and F. Sciortino, Dynamics in the Presence of Attractive Patchy Interactions, J. Phys. Chem. B 110, 8064 (2006).

[63] J. Russo, P. Tartaglia, and F. Sciortino, Reversible Gels of Patchy Particles: Role of the Calence, J. Chem. Phys. 131, 014504 (2009).

[64] L. Rovigatti, P. Šulc, Z. R. Istv, and F. Romano, A Comparison between Parallelization Approaches in Molecular Dynamics Simulations on GPUs, J. Comput. Chem. 36, 1 (2015).

[65] A. Clauset, C. R. Shalizi, and M. E. Newman, Power-Law Distributions in Empirical Data, SIAM Rev. 51, 661 (2009).

[66] F. A. Graybill, D. C. Boes, and A. M. Mood, Introduction to the Theory of Statistics (McGraw-Hill, New York, 1974).

[67] SCIPY Community, SCIPY.OPTIMIZE.MINIMIZE, https://docs .scipy.org/doc/scipy/reference/generated/scipy.optimize .minimize.html\#scipy.optimize.minimize, 2019.

[68] J. Leitão, J. Miotto, M. Gerlach, and E. G. Altmann, Is This Scaling Nonlinear?, R. Soc. Open Sci. 3, 150649 (2016).

Correction: A statement regarding the rescaling of $\lambda$ in Fig. 2(b) was missing from the caption and has been inserted. 